\title{
LEVI GEOMETRY \\ AND THE TANGENTIAL CAUCHY-RIEMANN EQUATIONS ON A REAL ANALYTIC SUBMANIFOLD OF $\mathrm{C}^{n}$ \\ BY
}

AL BOGGESS

\begin{abstract}
The relationship between the Levi geometry of a submanifold of $\mathbf{C}^{n}$ and the tangential Cauchy-Riemann equations is studied. On a real analytic codimension two submanifold of $\mathbf{C}^{n}$, we find conditions on the Levi algebra which allow us to locally solve the tangential Cauchy-Riemann equations (in most bidegrees) with kernels. Under the same conditions, we show that, locally, any CR-function is the boundary value jump of a holomorphic function defined on some suitable open set in $\mathbf{C}^{n}$. This boundary value jump result is the best possible result because we also show that there is no one-sided extension theory for such submanifolds of $\mathbf{C}^{n}$. In fact, we show that if $S$ is a real analytic, generic, submanifold of $\mathbf{C}^{n}$ (any codimension) where the excess dimension of the Levi algebra is less than the real codimension, then $S$ is not extendible to any open set in $\mathbf{C}^{n}$.
\end{abstract}

0. Introduction. Recently, there has been increasing interest in the relationship of geometric concepts, such as the Levi form of a hypersurface and the local solvability of the tangential Cauchy-Riemann equations. For example, Henkin [H] has shown that on a strictly pseudoconvex hypersurface, one can locally solve the tangential Cauchy-Riemann equations (in most bidegrees). His approach involved explicit integral kernels. In this paper we start such a program for higher codimension. We relate such concepts as the Levi form and the Levi algebra to the local solvability of the tangential Cauchy-Riemann equations on a real analytic, codimension two submanifold of $\mathbf{C}^{n}$. The geometric conditions we impose are in terms of strict pseudoconvexity of the Levi form (Definition 2.3) and the excess dimension of the Levi algebra (Definition 2.2). We represent the local solution to the tangential Cauchy-Riemann equations in terms of explicit kernels. We show (Theorem 5.1) that on a generic real analytic, strictly pseudoconvex, codimension two submanifold $S$ of $\mathrm{C}^{n}$ with excess dimension of $S$ equal to one, we can locally solve the tangential Cauchy-Riemann equations in bidegrees $(p, q)$ where $1 \leqslant q \leqslant n-3$. Although our emphasis is on codimension two, we indicate an inductive process for local solvability on higher codimension submanifolds of $\mathbf{C}^{n}$.

From the kernel approach we also get, for free, a local result (Theorem 5.15) about CR-functions on a codimension two submanifold, $S$, satisfying the hypothesis of Theorem 5.1. Locally, we carefully construct an open set $V$ in $\mathbf{C}^{n}$ with $\bar{V} \cap S \neq \varnothing$ such that a CR-function on $\bar{V} \cap S$ is the boundary value jump across $S$ of a

Received by the editors January 24, 1980 and, in revised form, February 9, 1981. 1980 Mathematics Subject Classification. Primary 35N15, 32F99.

(C)1982 American Mathematical Society 0002-9939/82/0000-1064/\$06.75 
holomorphic function defined on $V$. Related to this result, we prove that there is no one-sided extension theory for CR-functions on $S$. In fact, we show (Theorem 5.22) that on a real analytic, generic, submanifold of $\mathbf{C}^{n}$ (any codimension $\leqslant n$ ) where the excess dimension is less than the (real) codimension, there does not exist a fixed open set in $\mathbf{C}^{n}$ to which all $\mathbf{C R}$-functions extend as holomorphic functions. This means that the boundary value jump result (Theorem 5.15) is the best possible result for real analytic, codimension two submanifolds with excess dimension equal to one. In the real analytic case, Theorem 5.22 also provides a converse to the result of Hunt and Wells [HW] (see also Greenfield [Gr]) which roughly states that if $S$ is a generic submanifold with maximal excess dimension (i.e. excess dimension $=($ real $)$ codimension), then $S$ is extendible to an open set in $\mathbf{C}^{n}$.

The operators involved in the local solvability result (Theorem 5.1) are the boundary value jumps across $S$ of kernels which are defined on carefully chosen hypersurfaces which contain $S$. To find these hypersurfaces, we prove the following geometric result (Theorem 3.4), which is. of independent interest: A real analytic submanifold of real dimension $2 n-k$ with excess dimension equal to $k-p$, $0 \leqslant p \leqslant k$, is locally contained in the intersection of $p$ transverse real analytic, Levi flat hypersurfaces. A uniqueness part is also proven. An analogous result, without the uniqueness part, is also proven for $C^{\infty}$ submanifolds.

We have organized the paper as follows: In $\$ 2$ the geometric concepts such as the Levi form and the Levi algebra are explained from a Pfaffian system (differential forms) point of view. This point of view is somewhat different from the vector field approach introduced by Greenfield [Gr] and Hill and Taiani [HT], which is more standardly used. But the roots of our approach are actually more classical in nature, going back to Cartan [C]. Whenever possible, we relate the two points of view. Our approach only requires the most elementary concepts from Pfaffian systems, which we set down in $\$ 1$. $\S 3$ is devoted to our geometric result stated above. The essential tools we use are the real Frobenius theorem and Tomassini's result [T], which states that a real analytic CR-function on a real analytic submanifold is locally the restriction of a unique holomorphic function. In $\S 4$, we state some formal identities which relate a class of kernels to the tangential Cauchy-Riemann equations. Few proofs are given in this section, since this material is very thoroughly discussed in Harvey and Polking [HP]. Finally, we state and prove our local solvability result in $\$ 5$.

I would like to thank John Polking and Reese Harvey for useful discussions about the Pfaffian system approach to the Levi algebra. I would also like to thank Dan Burns. Discussions with him led, in particular, to the uniqueness part of Theorem 3.4.

1. Pfaffian systems. In this section, we describe the fundamental concepts of Pfaffian systems. For more details we refer the reader to Gardner [G].

Let $S$ be a $C^{\infty}$ manifold, and let $\mathcal{E}(S)$ be the ring of smooth functions on $S$. We let $T^{*}(S)$ (resp. $T(S)$ ) be the cotangent bundle (resp. tangent bundle) of $S$. If $U$ is an open set in $S$, then $\Gamma^{*}(U)$ (resp. $\Gamma(U)$ ) will denote the space of smooth sections of $T^{*}(S)($ resp. $T(S))$ over $U$. 
1.1. Definition. A Pfaffian system over an open set $U \subset S$ is an $\mathcal{E}(S)$ submodule of $\Gamma^{*}(U)$.

Dually, we define:

1.2. Definition. A vector field system over an open set $U \subset S$ is an $\mathcal{E}(S)$ submodule of $\Gamma(U)$.

Let 9 be a Pfaffian system over an open set $U \subset S$; then we define the annihilator of $q, \stackrel{9}{q}$, to be the set of vector fields on $U$ which are annihilated by $q$, i.e.

$$
\stackrel{\AA}{q}=\{X \in \Gamma(S) ;(\eta, X)=0 \text { for each } \eta \in \mathscr{q}\} \text {. }
$$

We have used the notation $(\eta, X)$ to be the duality pairing between the one form $\eta$ and the vector field $X$.

In what follows, all Pfaffian systems and vector field systems are assumed to have constant dimension. If $G$ is a Pfaffian system of dimension $l$ and if $u_{1}, \ldots, u_{l} \in \mathcal{G}$ generate $\mathcal{G}$ over $\mathcal{E}(U)$, then we often write $\mathscr{G}=\operatorname{sp}\left\{u_{1}, \ldots, u_{l}\right\}$.

1.3. Definition. Let $\mathscr{G}$ be a Pfaffian system over an open set $U \subset S . \mathscr{G}$ is said to be integrable if for each $\eta \in \mathcal{G}$ we have $d_{S} \eta \in \mathcal{G} \wedge \Gamma^{*}(U)$, where $d_{S}: \Lambda^{p} T^{*}(S) \rightarrow$ $\Lambda^{p+1} T^{*}(S)$ is the exterior derivative operator on $S$.

It is often useful to understand how the concepts for Pfaffian systems translate for vector fields. For example, a Pfaffian system $G$ is integrable if and only if $\stackrel{9}{q}$ is involutive, i.e. $\left[X_{1}, X_{2}\right] \in \stackrel{\circ}{9}$ whenever $X_{1}, X_{2} \in \stackrel{\circ}{9}$. This follows immediately from the equation

$$
\left(d_{s} \eta, X_{1} \wedge X_{2}\right)=\left(\eta,\left[X_{1}, X_{2}\right]\right) ; \quad \eta \in \mathscr{q} ; X_{1}, X_{2} \in \stackrel{\AA}{9} .
$$

The important theorem about integrable Pfaffian systems is the following Froebenius theorem (see [F, p. 97]):

1.5. Theorem. Suppose $S$ is a smooth (resp. real analytic) manifold. Suppose 9 is a smooth (resp. real analytic) integrable Pfaffian system of (real) dimension l defined in a neighborhood of a point $x_{0} \in S$. Then, locally, there exist smooth (resp. real analytic) functions $u_{1}, \ldots, u_{l}: S \rightarrow \mathbf{R}$ with $u_{1}\left(x_{0}\right)=\cdots=u_{l}\left(x_{0}\right)=0 ; d_{S} u_{1}\left(x_{0}\right)$ $\wedge \cdots \wedge d_{s} u_{l}\left(x_{0}\right) \neq 0$ and

$$
g=\operatorname{sp}\left\{d_{s} u_{1}, \ldots, d_{s} u_{l}\right\} .
$$

Let $\mathcal{G}$ be a Pfaffian system over an open set $U \subset S$. The exterior derivative $d_{S}$ : $g \rightarrow \Lambda^{2} \Gamma^{*}(U)$ is not an $\mathcal{E}(U)$ module homomorphism. To remedy this we let $\delta_{1}$ : $\oint \rightarrow \Lambda^{2} \Gamma^{*}(U) / \Gamma^{*}(U) \wedge q$ be the following composition:

$$
\stackrel{d_{s}}{\rightarrow} \Lambda^{2} \Gamma^{*}(U) \stackrel{\pi}{\rightarrow} \Lambda^{2} \Gamma^{*}(U) / \Gamma^{*}(U) \wedge q
$$

where $\pi$ denotes natural projection. It is clear that $\delta_{1}$ is an $\mathcal{E}(U)$ module homomorphism. The kernel of $\delta_{1}$ is an $\mathcal{E}(U)$ submodule of $\mathscr{G}$ which we denote by $\mathscr{G}_{1}$. This submodule is often called the first derived system of 9 . We inductively define $\delta_{r}$ : $g_{r-1} \rightarrow \Lambda^{2} \Gamma^{*}(U) / \Gamma^{*}(U) \wedge g_{r-1}$ as the composition

$$
g_{r-1} \stackrel{d_{s}}{\rightarrow} \Lambda^{2} \Gamma^{*}(U) \stackrel{\pi_{r-1}}{\rightarrow} \Lambda^{2} \Gamma^{*}(U) / \Gamma^{*}(U) \wedge g_{r-1},
$$


where again $\pi_{r-1}$ denotes natural projection. The $r$ th derived system $\mathscr{Q}_{r} \subset \mathscr{Q}_{r-1}$ is then defined to be the kernel of $\delta_{r}$. We have the exact sequence:

$$
0 \rightarrow g_{r} \rightarrow g_{r-1} \stackrel{\delta_{r}}{\rightarrow} \delta_{r}\left(g_{r-1}\right) \rightarrow 0
$$

where $\delta_{r}\left(G_{r-1}\right)$ is the image of $\mathscr{G}_{r-1}$ in $\Lambda^{2} \Gamma^{*}(U) / \Gamma^{*}(U) \wedge \mathscr{G}_{r-1}$ under $\delta_{r}$. Clearly, we have

$$
\cdots \subset g_{r} \subset g_{r-1} \subset \cdots \subset q_{1} \subset \Phi_{0}=9,
$$

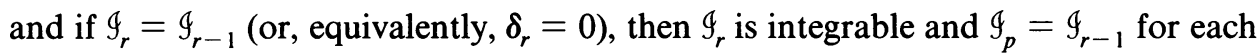
$p \geqslant r$. There is a smallest integer $N$, called the derived length of $\mathscr{G}$, such that $q_{N+p}=q_{N}$ for each $p \geqslant 0$. We shall often write $\oint_{\text {int }}$ for $\oint_{N}$. It is clear from the definitions that $\oint_{\text {int }}$ is the largest integrable system of $\mathscr{G}$ and that

$$
g_{\text {int }}=\bigcap_{l=1}^{\infty} g_{l} \text {. }
$$

From the vector field point of view, we have $\dot{q}^{\circ} \subset \dot{q}_{1} \subset \ldots \subset \stackrel{9}{q}_{r} \subset \ldots$, and $\dot{q}_{r}$ is spanned by $\stackrel{\circ}{r-1}_{r-1}$ and all elements which are $r$ th Lie brackets of elements in $\stackrel{\circ}{9}$. We also have

$$
\dot{\Phi}_{\text {int }}=\bigcup_{l=1}^{\infty} \stackrel{\circ}{q}_{l}
$$

and $\stackrel{\circ}{\mathrm{g}}_{\text {int }}$ is the vector field system generated (over $\mathcal{E}(U)$ ) by $\stackrel{\circ}{\mathrm{g}}$ and all Lie brackets of $\stackrel{\circ}{q}$, i.e. $\stackrel{\mathscr{G}}{\text { int }}_{\text {is }}$ the Lie algebra of $\stackrel{\AA}{9}$.

2. The Levi form and Levi algebra. In this section we explain the Levi form and Levi algebra in terms of a particular Pfaffian system. This point of view is useful when the information that is desired from the Levi form is in terms of differential forms (as opposed to vector fields).

We shall make use of the standard pointwise Euclidean metric for $\Lambda^{r} T^{*}\left(\mathbf{R}^{m}\right)$, which we denote by $\langle\phi, \psi\rangle_{x}, \psi, \phi \in \Lambda_{x}^{r} T^{*}\left(\mathbf{R}^{m}\right)$. To emphasize that this is a real (vs. complex hermitian) inner product, we may write $\langle\phi, \psi\rangle_{x}^{\mathbf{R}}$.

Now, suppose $S$ is a generic submanifold of $\mathbf{C}^{n}$ of (real) dimension $2 n-k$. Let $H^{*}(S)=T^{*}(S) \cap J T^{*}(S)$ be the holomorphic cotangent bundle of $S$, where $J$ : $T^{*}\left(\mathbf{R}^{2 n}\right) \rightarrow T^{*}\left(\mathbf{R}^{2 n}\right)$ is the complex structure on $T^{*}\left(\mathbf{R}^{2 n}\right)$. Since $S$ is generic, $H^{*}(S)$ is a Pfaffian system of minimal dimension, i.e. the dimension of each fiber is $2(n-k)$. Let 9 be the orthogonal complement of $H^{*}(S)$ in $T^{*}(S)$. Clearly, $T^{*}(S)=H^{*}(S) \oplus$ 9 . The Pfaffian system $\mathscr{G}$ is of real dimension $k$ and $\mathscr{G}$ is sometimes called the totally real subbundle of $T^{*}(S)$. In fact, if $S$ is locally defined by $S=\left\{x \in \mathbf{C}^{n} ; r_{1}(x)=\right.$ $\left.\cdots=r_{k}(x)=0\right\}$ where $r_{1}, \ldots, r_{k}: \mathbf{C}^{n} \rightarrow \mathbf{R}$ are smooth functions with $d r_{1} \wedge \cdots \wedge d r_{k}$ $\neq 0$ on $S$, then locally:

$$
\begin{aligned}
\mathscr{G} & =\operatorname{sp}\left\{\left.J d r_{1}\right|_{s}, \ldots,\left.J d r_{k}\right|_{s}\right\} \\
& =\operatorname{sp}\left\{\left.d^{c} r_{1}\right|_{s}, \ldots,\left.d^{c} r_{k}\right|_{s}\right\} \quad\left(d^{c}=J \circ d=i(\partial-\bar{\partial})\right) .
\end{aligned}
$$

2.1. Definition. If $S$ is generic, then we say that $S$ is regular if $\mathscr{G}$ and each of its derived systems have constant rank on $S$. 
We shall only consider regular submanifolds in this work. This allows us to think of 9 and its derived systems as subbundles of $T^{*}(S)$.

2.2. Definition. The $l$ th Levi form of $S$ is the map $\delta_{l}: \mathscr{G}_{l-1} \rightarrow \Lambda^{2} \Gamma^{*}(S) / \mathscr{G}_{l-1} \wedge$ $\Gamma^{*}(S)$, where we have used the notation from $\S 1$.

Recall that if $\delta_{l}=0$ then $\Phi_{l-1}$ is an integrable subbundle of $\oint_{\text {. So the Levi forms }}$ on $S$ measure the integrability of the subbundles of the totally real subbundle $9 \subset T^{*}(S)$.

Since $\Lambda^{2} T^{*}(S) / \mathscr{G} \wedge T^{*}(S)$ can be identified with $\Lambda^{2} H^{*}(S)$, it is clear that $\delta_{1}\left(\left.J d r_{j}\right|_{S}\right)$ is just the orthogonal projection of $\left.d\left(J d r_{j}\right)\right|_{S}=\left.d d^{c} r_{j}\right|_{S}=\left.2 i \bar{\partial} \partial r_{j}\right|_{S}$ onto $\Lambda^{2} H^{*}(S)=\Lambda^{2}\left(T^{*}(S) / \mathcal{G}\right)$. In general, if $\eta \in \mathscr{G}$ then $\delta_{1}(\eta)$ is just the orthogonal projection of $\left.d \tilde{\eta}\right|_{S}$ onto $\Lambda^{2} H^{*}(S)$, where $\tilde{\eta}$ is any ambient extension of $\eta$. Similarly, $\delta_{l}(\eta)$ is just the orthogonal projection of $\left.d \tilde{\eta}\right|_{S}$ onto $\Lambda^{2}\left(T^{*}(S) / q_{l-1}\right)$.

2.3. Definition. Suppose $S$ is regular. The excess dimension of the Levi algebra of $S$, denoted by ex $\operatorname{dim}(S)$, is the real dimension of the quotient $G / G_{\text {int }}$, where $\Phi_{\text {int }}$ is the maximal integrable subbundle of $G$.

From $\S 1$ it is clear that $g / \mathscr{G}_{\text {int }} \simeq \dot{q} / \dot{\Phi}_{\text {int }}$. The space $\stackrel{\leftrightarrow}{\text { int }}_{\text {is }}$ is the Levi algebra generated by $\stackrel{9}{q}=$ the holomorphic tangent bundle of $S$. So from a vector field point of view, the quantity ex $\operatorname{dim}(S)$ measures the dimension of the Levi algebra of $S$ relative to the dimension of the holomorphic tangent bundle of $S$.

It is clear that $0 \leqslant \operatorname{ex} \operatorname{dim}(S) \leqslant k=\operatorname{codim}(S)$. A submanifold $S$ of $\mathbf{C}^{n}$ is said to be Levi flat if ex $\operatorname{dim}(S)=0$ or, equivalently, if $\mathscr{G}$ is integrable.

2.4. Definition. Suppose $S$ is a submanifold of $\mathbf{C}^{n}$ and $x_{0} \in S . S$ is said to be strictly pseudoconvex at $x_{0}$ if there exists an $\eta \in \mathscr{Q}$ and a constant $C>0$ such that

$$
\left\langle\delta_{1}(\eta),\left.\omega \wedge J \omega\right|_{x_{0}}\right\rangle_{x_{0}}^{\mathbf{R}} \geqslant C|\omega|_{x_{0}}^{2}
$$

for each $\omega \in H_{x_{0}}^{*}(S)$.

This inequality is equivalent to the following inequality:

$$
\frac{i}{2}\langle d \tilde{\eta}, W \wedge \bar{W}\rangle_{x_{0}}^{\mathbf{C}} \geqslant C|W|_{x_{0}}^{2},
$$

where $W=\omega-i J \omega$ is in the complexified holomorphic cotangent bundle, $\langle,\rangle_{x_{0}}^{\mathbf{C}}$ denotes the complex hermitian inner-product on forms, and $\tilde{\eta}$ is any ambient extension of $\eta$.

For example, suppose $S$ is a real hypersurface in $\mathbf{C}^{n}$, locally defined by $S=\{x \in$ $\left.\mathbf{C}^{n} ; r(x)=0\right\}$ where $r: \mathbf{C}^{n} \rightarrow \mathbf{R}$ is smooth with $d r \neq 0$ on $S$. Clearly $\mathscr{G}=\operatorname{sp}\left\{\left.J d r\right|_{S}\right\}$ $=\operatorname{sp}\left\{\left.d^{c} r\right|_{S}\right\}$ and ex $\operatorname{dim}(S) \leqslant 1 . S$ is strictly pseudoconvex at a point $x_{0} \in S$ if either $r$ or $-r$ satisfies

$$
\langle\partial \bar{\partial} r, W \wedge \bar{W}\rangle_{x_{0}}^{\mathbf{c}} \geqslant C|W|^{2}
$$

for any $W$ which is in the complexified holomorphic cotangent space of $S$ at $x_{0}$. Written out in coordinates, $W=\left(w_{1}, \ldots, w_{n}\right)$; this is equivalent to

$$
\sum_{j, k=1}^{n} \frac{\partial^{2} r}{\partial z_{j} \overline{\partial z}_{k}}\left(x_{0}\right) w_{j} \bar{w}_{k} \geqslant C|W|^{2}
$$

provided $\sum_{j=1}^{n}\left(\partial r / \partial z_{j}\right) w_{j}=0$. 
So the above concepts about the Levi form agree with the classical concepts about the Levi form on a hypersurface. Moreover, in higher codimension, the above concepts also agree with those concepts defined from a vector field point of view; see Greenfield [Gr] and Hill and Taiani [HT]. One must, however, use (1.4) to go from the point of view described here to the vector field point of view.

3. Levi geometry in higher codimension. Suppose $S$ is a generic submanifold of $\mathbf{C}^{n}$. The real cotangent bundle of $S, T^{*}(S)=T^{*}(S) \otimes 1$, can be imbedded into its complexification $\mathrm{C} T^{*}(S)=T^{*}(S) \otimes_{\mathbf{R}} \mathrm{C}$. We let $\mathrm{CH}^{*}(S)$ and $\mathrm{C} 9$ denote the complexified holomorphic cotangent bundle and the complexified totally real subbundle of $S$, respectively. Using the complex hermitian innerproduct, $\mathrm{CH}^{*}(S)$ orthogonally decomposes into $\mathrm{CH}^{*}(S)=H^{1,0}(S) \oplus H^{0,1}(S)$ where $H^{1,0}(S)$ and $H^{0,1}(S)$ are, respectively, the $+i$ and $-i$ eigenspaces of the complex structure map $J: \mathrm{CH}^{*}(S) \rightarrow$ $\mathrm{C} H^{*}(S)$. Thus, $\mathrm{CT}^{*}(S)$ has the orthogonal splitting

$$
\mathrm{C} T^{*}(S)=H^{1,0}(S) \oplus H^{0,1}(S) \oplus \mathbf{C} 9 \text {. }
$$

We make the following definition of a CR-function on $S$, which is useful for our purposes.

3.2. Definition. Suppose $V \subset S$ is an open set. A smooth function $f: V \rightarrow \mathbf{C}$ is called a CR-function on $V$ if $\left(d_{s} f\right)(x)$ is orthogonal to $H_{x}^{0,1}(S)$ for each $x \in V$.

If $S$ is locally defined by $S=\left\{x \in \mathbf{C}^{n} ; r_{1}(x)=\cdots=r_{k}(x)=0\right\}$, where each $r_{j}$ : $\mathbf{C}^{n} \rightarrow \mathbf{R}$ is smooth and $d r_{1} \wedge \cdots \wedge d r_{k} \neq 0$ on $S$, then the above definition is equivalent to $\bar{\partial} \tilde{f} \wedge \bar{\partial} r_{1} \wedge \cdots \wedge \bar{\partial} r_{k}=0$ on $V$, where $\tilde{f}$ is any smooth ambient extension of $f$. The fact we need about real analytic CR-functions is the following result due to Tomassini [T] (see also Greenfield [Gr, p. 310]).

3.3. TheOREM. Suppose $S$ is a real analytic generic submanifold. Suppose $f$ is a real analytic $C R$-function on some nonempty open subset $V \subset S$. Then $f$ is the restriction to $V$ of a unique holomorphic function defined on some neighborhood of $V$ in $\mathbf{C}^{n}$.

The purpose of this section is to use Tomassini's theorem to prove the following geometric result:

3.4. THEOREM. Suppose $S$ is a real analytic, regular submanifold of $\mathbf{C}^{n}$ of real dimension $2 n-k$. Suppose ex $\operatorname{dim}(S)=k-p, 0 \leqslant p \leqslant k$, and suppose $x_{0} \in S$. Then there exist $p$ transverse, real analytic, Levi flat hypersurfaces $N_{1}, \ldots, N_{p}$ which are Levi flat near $x_{0}$ with $S \subset N_{1} \cap \cdots \cap N_{p}$. Moreover, there exist functions $F_{1}, \ldots, F_{p}$ which are holomorphic near $x_{0}$, such that $N_{i}=\left\{x \in \mathbf{C}^{n} ; \operatorname{Re} F_{i}(x)=0\right\}, 1 \leqslant i \leqslant p$. The submanifold $N_{1} \cap \cdots \cap N_{p}$ is a real analytic Levi flat submanifold of codimension $p$ which is unique in the following sense. If $\tilde{N}_{1}, \ldots, \tilde{N}_{p}$ are transverse Levi flat, real analytic hypersurfaces with $S \subset \tilde{N}_{1} \cap \cdots \cap \tilde{N}_{p}$, then there is a neighborhood $U$ of $x_{0}$ in $\mathrm{C}^{n}$ with $N_{1} \cap \cdots \cap N_{p} \cap U=\tilde{N}_{1} \cap \cdots \cap \tilde{N}_{p} \cap U$.

Proof. Using the notation from $\S \S 1$ and 2 , we let $\delta_{l}: q_{l-1} \rightarrow \Lambda^{2} \Gamma^{*}(S) / q_{l-1} \wedge$ $\Gamma^{*}(S)$ be the $l$ th Levi form on $S$ and $\oint_{l}=\operatorname{ker} \delta_{l}$. The assumptions on $S$ imply that 
$q_{\text {int }}=\bigcap_{l=1}^{\infty} q_{l}$ is the maximal integrable subbundle of $G$ of real dimension $p$. (Note that $k-p=\operatorname{ex} \operatorname{dim}(S)=\operatorname{dim}\left(G / \oint_{\text {int }}\right) \Rightarrow \operatorname{dim} \oint_{\text {int }}=p$.)

The idea is to find $v_{1}, \ldots, v_{p}: S \rightarrow \mathbf{R}$ which are real analytic and CR near $x_{0}$, with $\operatorname{sp}\left\{d_{s} v_{1}, \ldots, d_{s} v_{p}\right\}=g_{\text {int }}$. Then we shall use Tomassini's theorem to find $F_{1}, \ldots, F_{p}$ which are holomorphic near $x_{0}$ with $\left.F_{j}\right|_{s}=i v_{j}, 1 \leqslant j \leqslant p$. The hypersurfaces $N_{j}=$ $\left\{x \in \mathbf{C}^{n} ; \operatorname{Re} F_{j}(x)=0\right\}, 1 \leqslant j \leqslant p$, will be the desired Levi flat hypersurfaces (note: $i=\sqrt{-1}$ ).

Now, $\mathscr{G}_{\text {int }}$ is an integrable, real analytic subbundle of $\mathscr{G}$ (i.e. $d_{s} g_{\text {int }} \subset g_{\text {int }} \wedge T^{*}(S)$ ). By the real Froebenius theorem, there exist $v_{1}, \ldots, v_{p}: S \rightarrow \mathbf{R}$ which are real analytic near $x_{0}$ such that $\operatorname{sp}\left\{d_{S} v_{1}, \ldots, d_{s} v_{p}\right\}=g_{\text {int }}$ near $x_{0}$, and $v_{1}\left(x_{0}\right)=\cdots=v_{p}\left(x_{0}\right)=0$. In particular, $d_{S} v_{1} \wedge \cdots \wedge d_{S} v_{p} \neq 0$. From the point of view of vector fields, recall that $\stackrel{\circ}{\text { int }}_{\text {int }}=\left\{X \in T(S) ;(\eta, X)=0\right.$ for each $\left.\eta \in \mathscr{G}_{\text {int }}\right\}$. The integrability of $q_{\text {int }}$ implies that $\dot{g}_{\text {int }}$ is involutive, and the functions $v_{1}, \ldots, v_{p}$ found above satisfy $X\left(v_{j}\right)=0$ for each $X \in \stackrel{\circ}{\text { int }}_{\text {and }} 1 \leqslant j \leqslant p$.

Since $d_{S} v_{j} \in \mathscr{G}_{\text {int }} \subset \mathcal{G} \subset \mathrm{C} \mathscr{G}$, each $v_{j}$ is a CR-function on $S$ near $x_{0}$ by Definition 3.2 and by the orthogonal decomposition given in (3.1). So, by Theorem $3.3, i v_{j}$ is the restriction of a function $F_{j}\left(\left.F_{j}\right|_{s}=i v_{j}\right)$ which is holomorphic in some neighborhood in $\mathbf{C}^{n}$ containing $x_{0}$. Let $R_{j}=\operatorname{Re} F_{j}$ and $I_{j}=\operatorname{Im} F_{j}, 1 \leqslant j \leqslant p$. The hypersurfaces $N_{j}=\left\{x \in \mathbf{C}^{n} ; R_{j}(x)=0\right\}, 1 \leqslant j \leqslant p$, are clearly real analytic and Levi flat. Since each $F_{j}$ is purely imaginary on $S$, clearly $S \subset N_{1} \cap \cdots \cap N_{p}$. To ensure that $N_{1}, \ldots, N_{p}$ are transverse, we only need to check that $d R_{1} \wedge \cdots \wedge d R_{p} \neq 0$ at $x_{0}$. But if $d R_{1} \wedge \cdots \wedge d R_{p}=0$ at $x_{0}$, then certainly $d I_{1} \wedge \cdots \wedge d I_{p}=0$ at $x_{0}$, because the Cauchy-Riemann equations imply that $d I_{j}=-J d R_{j}$. Since $\left.I_{j}\right|_{s}=v_{j}$, then $\left\{d I_{1}\right.$ $\wedge \cdots \wedge d I_{p}=0$ at $\left.x_{0}\right\} \Rightarrow\left\{d_{s} v_{1} \wedge \cdots \wedge d_{S} v_{p}=0\right.$ at $\left.x_{0}\right\}$, which is a contradiction to the choice of $v_{1}, \ldots, v_{p}$. Therefore, $d R_{1} \wedge \cdots \wedge d R_{p} \neq 0$ at $x_{0}$ as desired.

To prove uniqueness, suppose $\tilde{F}_{j}=\left(\tilde{R}_{j}+i \tilde{I}_{j}\right), 1 \leqslant j \leqslant p$, are holomorphic functions near $x_{0}$, with $S \subset\left\{x \in \mathbf{C}^{n} ; \tilde{R}_{1}(x)=\cdots=\tilde{R}_{p}(x)=0\right\}$ and $d \tilde{R}_{1} \wedge \cdots \wedge d \tilde{R}_{p}$ $\neq 0$ on $S$. Let $\tilde{v}_{j}=\left.\tilde{I}_{j}\right|_{s}$. By adding a purely imaginary number to each $\tilde{F}_{j}$, we may assume $\tilde{v}_{j}\left(x_{0}\right)=0$. Clearly, $d_{S} \tilde{v}_{1} \wedge \cdots \wedge d_{S} \tilde{v}_{p} \neq 0$ on $S$ near $x_{0}$. We claim that

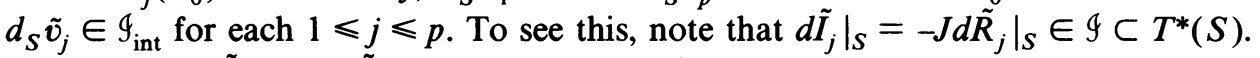
Therefore $d_{s}\left\{\left.\tilde{I}_{j}\right|_{s}\right\}=\left.d \tilde{I}_{j}\right|_{s}$, and so $d_{s} \tilde{v}_{j} \in 9$. Since $d_{S}\left(d_{s} \tilde{v}_{j}\right)=0$, it is clear that $\delta_{l}\left(d_{s} \tilde{v}_{j}\right)=0$ for each $l \geqslant 1$ and each $1 \leqslant j \leqslant p$. Therefore $d_{s} \tilde{v}_{j} \in \cap_{l=1}^{\infty} q_{l}=q_{\text {int }}$, for each $1 \leqslant j \leqslant p$, which proves our claim.

To sum up, we have the real analytic functions $v_{1}, \ldots, v_{p}$ and $\tilde{v}_{1}, \ldots, \tilde{v}_{p}$ with $\operatorname{sp}\left\{d_{s} v_{1}, \ldots, d_{s} v_{p}\right\}=g_{\text {int }}=\operatorname{sp}\left\{d_{s} \tilde{v}_{1}, \ldots, d_{s} \tilde{v}_{p}\right\}$. This means that each $\tilde{v}_{j}$ is a constant function on the level sets $V_{t}=\left\{x \in S ; v_{1}(x)=t_{1}, \ldots, v_{p}(x)=t_{p}\right\}$ for each $t=\left(t_{1}, \ldots, t_{p}\right) \in \mathbf{R}^{p}$ near the origin. Conversely, each $v_{j}$ is a constant function on the level sets $\tilde{V}_{t}=\left\{x \in S ; \tilde{v}_{1}(x)=t_{1}, \ldots, \tilde{v}_{p}(x)=t_{p}\right\}$. Therefore, there exist functions $f, g: \mathbf{R}^{p} \rightarrow \mathbf{R}^{p}$ which are real analytic near the origin such that $\tilde{v}=\left(\tilde{v}_{1}, \ldots, \tilde{v}_{p}\right)$ $=f\left(v_{1}, \ldots, v_{p}\right)$ and $v=\left(v_{1}, \ldots, v_{p}\right)=g\left(\tilde{v}_{1}, \ldots, \tilde{v}_{p}\right)$. If $f_{j}$ is the $j$ th component function of $f, 1 \leqslant j \leqslant p$, then $f_{j}$ has a power series expansion

$$
\tilde{v}_{j}=f_{j}\left(v_{1}, \ldots, v_{p}\right)=\sum_{\alpha=\alpha_{1} \cdots \alpha_{p}} a_{\alpha}^{j} v^{\alpha} \quad\left(a_{\alpha}^{j} \in \mathbf{R}\right)
$$


which is valid in some neighborhood of the origin. We claim that

$$
\begin{aligned}
\tilde{F}_{j} & =i f_{j}\left(-i F_{1}, \ldots,-i F_{p}\right) \\
& =i \sum_{\alpha=\alpha_{1}, \ldots, \alpha_{p}} a_{\alpha}^{j}\left(-i F_{1}\right)^{\alpha_{1}} \cdots\left(-i F_{p}\right)^{\alpha_{p}}
\end{aligned}
$$

near $x_{0}$. To see this, we note that both $\tilde{F}_{j}$ and $i f_{j}\left(-i F_{1}, \ldots,-i F_{p}\right)$ are holomorphic functions near $S$ which equal $i \tilde{v}_{j}$ on $S$. Therefore, $\tilde{F}_{j}=i f_{j}\left(-F_{1}, \ldots,-i F_{p}\right)$ by the uniqueness part of Theorem 3.3.

We now compute

$$
\begin{aligned}
\tilde{R}_{j} & =\operatorname{Re}\left\{i \sum_{\alpha=\alpha_{1} \cdots \alpha_{p}} a_{\alpha}^{j}(-i)^{|\alpha|}\left(R_{1}+i I_{1}\right)^{\alpha_{1}} \cdots\left(R_{p}+i I_{p}\right)^{\alpha_{p}}\right\} \\
& =-\sum_{\alpha=\alpha_{1}, \ldots, \alpha_{p}} a_{\alpha}^{j} \operatorname{Im}\left\{(-i)^{|\alpha|}\left(R_{1}+i I_{1}\right)^{\alpha_{1}} \cdots\left(R_{p}+i I_{p}\right)^{\alpha_{p}}\right\}
\end{aligned}
$$

where $|\alpha|=\alpha_{1}+\cdots+\alpha_{p}$. First we note that the constant term of this power series vanishes because $\tilde{R}_{j}=0$ on $S$. Next, we note that the terms of this power series which just involve $I_{1}, \ldots, I_{p}$ are

$$
-\sum_{\alpha=\alpha_{1} \cdots \alpha_{p}} a_{\alpha}^{j} \operatorname{Im}\left\{(-i)^{|\alpha|}(i)^{|\alpha|} I_{1}^{\alpha_{1}} \cdots I_{p}^{\alpha_{p}}\right\},
$$

which vanish. Therefore, all the terms in the power series for $\tilde{R}_{j}$ involve at least one factor of one of the functions $R_{q}, 1 \leqslant q \leqslant p$. In particular, there exists a neighborhood $U$ of $x_{0}$ in $\mathbf{C}^{n}$ (governed by the domain of convergence of $f$ ) such that

$$
\left\{x \in U ; R_{1}(x)=\cdots=R_{p}(x)=0\right\} \subset\left\{x \in U ; \tilde{R}_{1}(x)=\cdots=\tilde{R}_{p}(x)=0\right\} .
$$

The above argument can be repeated for $g$ to get the reverse inclusion. This proves our uniqueness assertion. Q.E.D.

REMARK. An analogous result in the $C^{\infty}$ category is also valid. Namely, we have

3.4'. THeOREM. Suppose $S$ satisfies the hypothesis of Theorem 3.4, except we only assume that $S$ is smooth. Then, locally, there exist hypersurfaces $N_{j}=\left\{x \in \mathbf{C}^{n}\right.$; $\left.r_{j}(x)=0\right\}$ where each $r_{j}: \mathbf{C}^{n} \rightarrow \mathbf{R}$ is smooth, $1 \leqslant j \leqslant p$, with $d r_{1} \wedge \cdots \wedge d r_{p} \neq 0$ on $S$ such that $S \subset N_{1} \cap \cdots \cap N_{p}$; and furthermore, for each $1 \leqslant j \leqslant p, \partial \bar{\partial} r_{j}$ vanishes to infinite order at $S$.

The proof for Theorem 3.4 is also valid for Theorem 3.4' except that instead of using Tomassini's result (Theorem 3.3), we use the following result due to Nirenberg and Wells [NW] and also [N]: A smooth CR-function on a smooth submanifold $S$ is locally the restriction to $S$ of a smooth function $f$ such that $\bar{\partial} f$ vanishes to infinite order on $S$.

In $\S 5$, we will need the following lemma, which shows that a smooth generic submanifold which is strictly pseudoconvex at a point is locally contained in a strictly pseudoconvex (real) hypersurface. 
3.5. Lemma. Suppose $S$ is a smooth generic submanifold of $\mathbf{C}^{n}$ of real dimension $2 n-k$. Let $x_{0} \in S$ and suppose $S$ is strictly pseudoconvex at $x_{0}$. Then there exists $a$ hypersurface $M=\left\{x \in \mathbf{C}^{n} ; \rho(x)=0\right\}, d \rho \neq 0, \rho: \mathbf{C}^{n} \rightarrow \mathbf{R}$ with $S \subset M$ and such that $\rho$ is strictly plurisubharmonic on a neighborhood of $x_{0}$ in $\mathbf{C}^{n}$.

Proof. As before, let $\mathcal{G}$ be the totally real subbundle of $T^{*}(S)$. Since $S$ is strictly pseudoconvex at $x_{0}$, there exists a one form $\eta \in \mathcal{G}$ of unit length and a positive constant $C$ such that

$$
\langle d \eta, \omega \wedge J \omega\rangle_{x_{0}}^{\mathbf{R}} \geqslant C|\omega|^{2}
$$

for $\omega \in H_{x_{0}}(S)$.

We can, locally, construct a smooth function $\rho_{1}: \mathbf{C}^{n} \rightarrow \mathbf{R}$, which vanishes on $S$ and satisfies $d \rho_{1}=-J \eta$ on $S$, near $x_{0}$. The function $\rho_{1}$ can be constructed as follows:

For $p \in S$, let $N_{p}^{*}(S)$ be the orthogonal complement (real Euclidean metric) to $T_{p}^{*}(S)$. Clearly $\operatorname{dim}_{\mathbf{R}} N_{p}(S)=k$ and $(J \eta)(p) \in N_{p}^{*}(S)$. Let $v_{2}, \ldots, v_{k} \in N^{*}(S)$ so that for each $p \in S$ near $x_{0},\left\{(J \eta)(p), v_{2}(p), \ldots, v_{k}(p)\right\}$ form an orthonormal basis for $N_{p}^{*}(S)$. Let $\phi: \mathbf{R}^{2 n-k} \rightarrow S$ be a local parameterization for $S$ near $x_{0}$. Consider $\Phi$ : $\mathbf{R}^{2 n-k} \times \mathbf{R}^{k-1} \rightarrow \mathbf{R}^{2 n}$,

$$
\Phi\left(x_{1} \cdots x_{2 n-k}, t_{2} \cdots t_{k}\right)=\phi(x)+t_{2} v_{2}(\phi(x))+\cdots+t_{k} v_{k}(\phi(x)) .
$$

Clearly $\Phi$ locally parameterizes a real hypersurface $M_{1}$ which contains $S$ (because $\Phi(x, 0)=\phi(x))$ and such that $(J \eta)(p)$ is orthogonal to $T_{p}^{*}\left(M_{1}\right)=T_{p}^{*}(S) \oplus$ $\operatorname{sp}\left\{v_{2}(p), \ldots, v_{k}(p)\right\}$. Let $\rho_{1}$ be a local defining function for $M_{1}$ normalized so that $d \rho_{1}$ has unit length. Then clearly $\left.d \rho_{1}\right|_{s}$ (or $-\left.d \rho_{1}\right|_{s}$ ) equals $-J \eta$.

The inequality in (3.6) can be written as $\left\langle\partial \bar{\partial} \rho_{1}, W \wedge \bar{W}\right\rangle_{x_{0}}^{\mathbf{C}^{n}} \geqslant C|W|^{2}$ for $W \in$ $H_{x_{0}}^{1,0}(S)$. This implies the complex Hessian of $\rho_{1}$ at $x_{0}$ has $n-k$ positive eigenvalues $\lambda_{1}, \ldots, \lambda_{n-k}$. From here on, we follow the standard argument (see Hunt and Wells [HW]) to obtain a strictly plurisubharmonic defining function. First, we find smooth functions $\rho_{2}, \ldots, \rho_{k}: \mathbf{C}^{n} \rightarrow \mathbf{R}$ such that, near $x_{0}, S=\left\{x \in \mathbf{C}^{n} ; \rho_{1}(x)=\cdots=\rho_{k}(x)\right.$ $=0\}$ and $d \rho_{1} \wedge \cdots \wedge d \rho_{k} \neq 0$ on $S$. Next, we make the biholomorphic change of variables given in Hunt and Wells [HW, p. 810]. That is, we can arrange new holomorphic coordinates for $\mathbf{C}^{n}, \tau=(z, w), z \in \mathbf{C}^{k}, w \in \mathbf{C}^{n-k}$, such that

$$
\rho_{j}(z, w)=\operatorname{Re} z_{j}+h_{j}(z, w), \quad 1 \leqslant j \leqslant k,
$$

where each $h_{j}(z, w)$ is a smooth function satisfying

$$
h_{j}(z, w)=\vartheta\left(|\tau|^{2}\right), \quad 1 \leqslant j \leqslant k,
$$

and, in particular,

$$
h_{1}(z, w)=\sum_{j=1}^{n-k} \lambda_{j}\left|w_{j}\right|^{2}+\vartheta\left(|\tau|^{3}\right) .
$$

We have used the notation $h=\vartheta\left(|\tau|^{q}\right)$ to mean that there are constants $C, \eta>0$ such that $|h(\tau)| \leqslant C|\tau|^{q}$ for $|\tau| \leqslant \eta$. Note that, in the new coordinates, $x_{0}=0$, $\eta=d y_{1}$, and $H_{0}^{1,0}(S)=\{0\} \times \mathbf{C}^{n-k} \simeq \mathbf{C}^{n-k}$ with coordinates $\left(w_{1}, \ldots, w_{n-k}\right)$. 
We now choose positive real constants $\gamma_{1}, \ldots, \gamma_{k}$ and make the following biholomorphic change of variables:

$$
\begin{aligned}
& z_{1}^{\prime}=z_{1}+\left(\gamma_{1} z_{1}^{2}+\cdots+\gamma_{k} z_{k}^{2}\right), \\
& z_{j}^{\prime}=z_{j}, 2 \leqslant j \leqslant k, \\
& w_{j}^{\prime}=w_{j}, 1 \leqslant j \leqslant n-k .
\end{aligned}
$$

It is clear that

$$
\operatorname{Re} z_{1}=x_{1}^{\prime}+\sum_{j=1}^{k} \gamma_{j}\left(\left(y_{j}^{\prime}\right)^{2}-\left(x_{j}^{\prime}\right)^{2}\right)+\vartheta\left(\left|\tau^{\prime}\right|^{3}\right)
$$

where we have set $x_{j}^{\prime}=\operatorname{Re} z_{j}^{\prime}, y_{j}^{\prime}=\operatorname{Im} z_{j}^{\prime}$ for $1 \leqslant j \leqslant k$. Therefore, in the new coordinates, we have

$$
\rho_{1}^{\prime}\left(z^{\prime}, w^{\prime}\right)=x_{1}^{\prime}+\sum_{j=1}^{k} \gamma_{j}\left(\left(y_{j}^{\prime}\right)^{2}-\left(x_{j}^{\prime}\right)^{2}\right)+\sum_{j=1}^{n-k} \lambda_{j}\left|w_{j}^{\prime}\right|^{2}+\vartheta\left(\left|\tau^{\prime}\right|^{3}\right)
$$

and

$$
\rho_{j}^{\prime}\left(z^{\prime}, w^{\prime}\right)=x_{j}^{\prime}+h_{j}^{\prime}\left(z^{\prime}, w^{\prime}\right) \text { for } 1 \leqslant j \leqslant k,
$$

with

$$
h_{j}^{\prime}\left(z^{\prime}, w^{\prime}\right)=\theta\left(\left|\tau^{\prime}\right|^{2}\right) .
$$

Now, on $S, x_{j}^{\prime}=-h_{j}^{\prime}\left(z^{\prime}, w^{\prime}\right)=\theta\left(\left|\tau^{\prime}\right|^{2}\right)$ for $1 \leqslant j \leqslant k$. Let $\rho_{1}^{\prime \prime}\left(z^{\prime}, w^{\prime}\right)$ be defined by setting $\left(x_{j}^{\prime}\right)^{2}=\left(h_{j}^{\prime}\left(z^{\prime}, w^{\prime}\right)\right)^{2}, 1 \leqslant j \leqslant k$, in the first sum appearing in (3.7). The function $\rho_{1}^{\prime \prime}$ also vanishes on $S$. Since $\left(h_{j}^{\prime}\left(\tau^{\prime}\right)\right)^{2}=\theta\left(\left|\tau^{\prime}\right|^{4}\right)$, we have

$$
\begin{aligned}
\rho_{1}^{\prime \prime}\left(z^{\prime}, w^{\prime}\right) & =x_{1}^{\prime}+\sum_{j=1}^{k} \gamma_{j}\left(y_{j}^{\prime}\right)^{2}+\sum_{j=1}^{n-k} \lambda_{j}\left|w_{j}^{\prime}\right|^{2}+\vartheta\left(\left|\tau^{\prime}\right|^{3}\right) \\
& =x_{1}^{\prime}-\frac{1}{2} \operatorname{Re}\left\{\sum_{j=1}^{k} \gamma_{j}\left(z_{j}^{\prime}\right)^{2}\right\}+\frac{1}{2}\left[\sum_{j=1}^{k} \gamma_{j}\left|z_{j}^{\prime}\right|^{2}\right]+\sum_{j=1}^{n-k} \lambda_{j}\left|w_{j}^{\prime}\right|^{2}+\vartheta\left(\left|\tau^{\prime}\right|^{3}\right) .
\end{aligned}
$$

Clearly, the complex hessian of $\rho_{1}^{\prime \prime}$ at the origin is positive definite with eigenvalues $\frac{1}{2} \gamma_{1}, \ldots, \frac{1}{2} \gamma_{k}, \lambda_{1}, \ldots, \lambda_{n-k}$. Strict plurisubharmonicity is an open condition; hence $\rho_{1}^{\prime \prime}$ is strictly plurisubharmonic in a neighborhood of the origin in $\mathbf{C}^{n}$.

Theorem 3.4 and Lemma 3.5 together imply the following result which we will need in $\$ 5$.

3.8. THEOREM. Suppose $S$ is a real analytic, regular submanifold of (real) dimension $2 n-k$. Suppose that ex $\operatorname{dim}(S)=k-p, 0 \leqslant p \leqslant k-1$, and that $S$ is strictly pseudoconvex at a point $x_{0} \in S$. Then there exist real analytic functions $r_{1}, \ldots, r_{p}, \rho$ : $\mathbf{C}^{n} \rightarrow \mathbf{R}$ and an open set $U$ in $\mathbf{C}^{n}$ containing $x_{0}$ with the following properties: (a) if $M=\left\{x \in \mathbf{C}^{n} ; \rho(x)=0\right\}$ and $N_{j}=\left\{x \in \mathbf{C}^{n} ; r_{j}(x)=0\right\}, 1 \leqslant j \leqslant p$, then $S \cap U \subset$ $M \cap N_{1} \cap \cdots \cap N_{p}$ and $d \rho \wedge d r_{1} \wedge \cdots \wedge d r_{p} \neq 0$ on $S \cap U$; (b) $\rho$ is strictly plurisubharmonic on $U$; (c) each $r_{j}$ is the real part of a holomorphic function on $U$.

Proof. Using Theorem 3.4, we may first find transverse hypersurfaces $N_{j}=\{x \in$ $\left.\mathrm{C}^{n} ; r_{j}(x)=0\right\}, 1 \leqslant j \leqslant p$, such that $S \subset N_{1} \cap \cdots \cap N_{p}$ and each $r_{j}$ is locally the real part of a holomorphic function. Then using Lemma 3.5, we may find a hypersurface 
$M=\left\{x \in \mathbf{C}^{n} ; \rho(x)=0\right\}$ such that $S \subset M$ and $\rho$ is strictly plurisubharmonic near $x_{0}$ in $\mathbf{C}^{n}$. Of course, the biholomorphisms occurring in the proof of Lemma 3.5 preserve the pluriharmonicity of each $r_{j}$. Thus, we need only show that $d \rho \wedge d r_{1}$ $\wedge \cdots \wedge d r_{p} \neq 0$ near $x_{0}$. As before, let $\oint_{\text {int }}=\cap_{l=1}^{\infty} q_{l}$, where

$$
q_{l}=\operatorname{ker} \delta_{l}: q_{l-1} \rightarrow \Lambda^{2} \Gamma^{*}(S) / q_{l-1} \wedge T^{*}(S) .
$$

Recall from the proof of Theorem 3.4 that $\operatorname{dim}_{\mathbf{R}} g_{\text {int }}=p$ and $g_{\text {int }}=$ $\operatorname{sp}\left\{\left.J d r_{1}\right|_{s}, \ldots,\left.J d r_{p}\right|_{s}\right\}$. If $d \rho \wedge d r_{1} \wedge \cdots \wedge d r_{p}=0$ at $x_{0}$, then

$$
J d \rho\left(x_{0}\right) \in \operatorname{sp}\left\{J d r_{1}\left(x_{0}\right), \ldots, J d r_{p}\left(x_{0}\right)\right\} .
$$

In particular, $\delta_{1}(J d \rho)\left(x_{0}\right)=0$. But since $\rho$ is strictly plurisubharmonic, clearly $\delta_{1}(J d \rho)\left(x_{0}\right) \neq 0$. Thus $d \rho \wedge d r_{1} \wedge \cdots \wedge d r_{p} \neq 0$ at $x_{0}$, as desired. Q.E.D.

4. Kernels and formal identities. In this section we describe the kernels we shall use and some formal identities which relate these kernels to the tangential Cauchy-Riemann equations. Much of this material is presented thoroughly in Harvey and Polking [HP], so we shall be brief. First, we need some more notation.

Let $\Lambda^{p, q}\left(\mathbf{C}^{n}\right)$ denote the bundle of forms on $\mathbf{C}^{n}$ of bidegree $p, q$. Smooth sections of $\Lambda^{p, q}\left(\mathbf{C}^{n}\right)$ over an open set $U \subset \mathbf{C}^{n}$ will be denoted by $\Gamma^{p, q}(U)$.

Suppose $S$ is a smooth generic submanifold of $\mathbf{C}^{n}$ of real dimension $2 n-k$, locally defined by $S=\left\{x \in \mathbf{C}^{n} ; \rho_{1}(x)=\cdots=\rho_{k}(x)=0\right\}$ with $d \rho_{1} \wedge \cdots \wedge d \rho_{k} \neq$ 0 near $S$. We let $I_{\rho}$ be the ideal in $\Lambda^{*}\left(\mathbf{C}^{n}\right)$ generated by $\bar{\partial} \rho_{1}, \ldots, \bar{\partial} \rho_{k}$. Since $S$ is generic, $I_{\rho}$ is a well-defined ideal of constant dimension defined over some set in $\mathbf{C}^{n}$ containing $S$. The restriction of this ideal to $S,\left.I_{\rho}\right|_{S}$, is called the ideal of complex normal forms on $S$. Using the complex hermitian metric, we let $I_{\rho}^{\perp}$ be the orthogonal complement of $I_{\rho}$ in $\Lambda^{*}\left(\mathbf{C}^{n}\right)$.

On $S$ we define $\left.\Lambda^{p, q}\left(\mathbf{C}^{n}\right)\right|_{S}$ to be the restriction of the bundle $\Lambda^{p, q}\left(\mathbf{C}^{n}\right)$ to $S$. An element in $\left.\Lambda^{p, q}\left(\mathbf{C}^{n}\right)\right|_{S}$ is an ambient $p, q$ form whose coefficients have been restricted to $S$. We also define

$$
\Lambda_{S}^{p, q}=\left\{\left.\left.\Lambda^{p, q}\left(\mathbf{C}^{n}\right)\right|_{S} \cap I_{\rho}^{\perp}\right|_{S}\right\},
$$

and we call this the bundle of complex tangential $(p, q)$ forms over $S$. For $V$ an open set in $S$, we let $\Gamma_{S}^{p, q}(V)$ be the space of smooth sections of $\Lambda_{S}^{p, q}$ over $V$ and we let $Q_{S}^{p, q}(V)$ be the space of sections in $\Gamma_{S}^{p, q}(V)$ with compact support in $V$.

There is a well-defined orthogonal projection map $T_{S}: \Lambda^{*}\left(\mathbf{C}^{n}\right) \rightarrow I_{\rho}^{\perp}$, and we shall often write $f_{T_{S}}$ for $T_{S}(f)$. If $\left.f \in \Lambda_{S}^{p, q}\left(\mathbf{C}^{n}\right)\right|_{S}$, then $f_{T_{S}} \in \Lambda_{S}^{p, q}$ and we call $f_{T_{S}}$ the complex tangential piece of $f$ along $S$. It must be kept in mind that if $f \in \Lambda^{*}\left(\mathbf{C}^{n}\right)$, then $f_{T_{S}}$ is defined on an open neighborhood of $S$ in $\mathbf{C}^{n}$.

The induced Cauchy-Riemann operator $\bar{\partial}_{S}: \Gamma_{S}^{p, q} \rightarrow \Gamma_{S}^{p, q+1}$ can now be defined as follows: Suppose $f \in \Gamma_{S}^{p, q}(V)$; choose $\tilde{f} \in \Gamma^{p, q}\left(\mathbf{C}^{n}\right)$ with $\tilde{f}_{T_{S}}=f$ on $V$; then $\bar{\partial}_{S} f=$ $\left(\left.\bar{\partial} \tilde{f}\right|_{S}\right)_{T_{S}} \in \Gamma_{S}^{p, q+1}(V)$. It can be easily verified that this definition is independent of the choice of ambient extension.

If $S$ is an orientable submanifold of $\mathbf{C}^{n}$ of real codimension $k$, then the current integration over $S^{\prime}$ is a current of degree $k$ defined by

$$
([S], \phi)_{\mathbf{C}^{n}}=\int_{S} \phi
$$


for $\phi \in \mathscr{Q}^{*}\left(\mathbf{C}^{n}\right)$. This current orthogonally splits into its bidegrees, i.e.

$$
[S]=\sum_{p+q=k}[S]^{p, q} .
$$

The most important piece of this splitting is $[S]^{0, k}$.

We have

$$
\left([S]^{0, k}, \phi\right)_{\mathbf{C}^{n}}=\int_{S} \phi
$$

where it is understood that only the piece of $\phi$ of bidegree $(n, n-k)$ is integrated. The important relation between $[S]^{0, k}$ and $\bar{\partial}_{S}$ is the following: if $f \in \Gamma_{S}^{p, q}$, then $\bar{\partial}_{S} f=g$ on $S$ if and only if $\bar{\partial} \tilde{f} \wedge[S]^{0, k}=\tilde{g} \wedge[S]^{0, k}$ where $\tilde{f}$ and $\tilde{g}$ are ambient extensions of $f$ and $g$, respectively (see [AB]).

Let $U$ be an open set in $\mathbf{C}^{n}$. Suppose $K(\zeta, z)$ is a form on $\mathbf{C}^{n} \times \mathbf{C}^{n}$ with smooth coefficients $S \times U$. Suppose $f \in \mathscr{D}_{S}^{p, q}(S)$. Then we let

$$
K\left([S]^{0, k} \wedge f\right)(z)=\int_{\zeta \in S} K(\zeta, z) \wedge \tilde{f}(\zeta)
$$

where $\tilde{f}$ is any ambient extension of $f$, and it is understood that only the piece of $K(\zeta, z) \wedge \tilde{f}(\zeta)$ of bidegree $(n, n-k)$ is integrated. It is clear that $K\left([S]^{0, k} \wedge f\right)(z)$ is a smooth form on $U$. In fact, if the bidegree of $K$ (in both $\zeta$ and $z$ ) is $(n, n-r)$, then $K: \mathscr{D}_{S}^{p, q} \rightarrow \Gamma^{p, q-r+k}(U)$. We also will need the following fundamental identity (see [HP, Theorem 2.8]):

$$
(\bar{\partial} K)\left([S]^{0, k} \wedge f\right)=\bar{\partial}\left\{K\left([S]^{0, k} \wedge f\right)\right\}+(-1)^{r+k} K\left([S]^{0, k} \wedge \bar{\partial}_{S} f\right),
$$

where the $\bar{\partial}$ on the left-hand side is in both the $\zeta$ and $z$ variables.

Now, suppose $M$ is a generic submanifold of $\mathbf{C}^{n}$ (later we will take $M$ to be a hypersurface). Let $V$ be an open subset of $M \times M$ with coordinates $(\zeta, z)$. Suppose $u^{j}(\zeta, z)=\left(u_{1}^{j}(\zeta, z), \ldots, u_{n}^{j}(\zeta, z)\right): V \rightarrow \mathbf{C}^{n}$ is a smooth mapping for $1 \leqslant j \leqslant N$. We adopt the following notation from [HP]:

$$
\begin{gathered}
u^{j}(\zeta, z) \cdot(\zeta-z)=\sum_{k=1}^{n} u_{k}^{j}(\zeta, z)\left(\zeta_{k}-z_{k}\right), \\
u^{j}(\zeta, z) \cdot d(\zeta-z)=\sum_{k=1}^{n} u_{k}^{j}(\zeta, z) d\left(z_{k}-z_{k}\right), \\
\bar{\partial}_{M} u^{j}(\zeta, z) \cdot d(\zeta-z)=\sum_{k=1}^{n} \bar{\partial}_{M} u_{k}^{j}(\zeta, z) \wedge d\left(\zeta_{k}-z_{k}\right),
\end{gathered}
$$

where $\bar{\partial}_{M}$ is taken in both variables $\zeta$ and $z$. We will use the following one forms:

$$
\alpha_{j}=\left[\frac{u^{j}(\zeta, z) \cdot d(\zeta-z)}{u^{j}(\zeta, z) \cdot(\zeta-z)}\right]_{T_{M \times M}}, \quad 1 \leqslant j \leqslant N .
$$

Each $\alpha_{j}$ is smooth on $V-A_{j}$ where

$$
A_{j}=\left\{(\zeta, z) \in V ; u^{j}(\zeta, z) \cdot(\zeta-z)=0\right\} .
$$


4.2. Proposition. For each integer $k \geqslant 1$, we have

$$
\alpha_{j} \wedge\left(\bar{\partial}_{M} \alpha_{j}\right)^{k}=\left[\frac{u^{j} \cdot d(\zeta-z)}{u^{j} \cdot(\zeta-z)}\right] \wedge\left[\frac{\bar{\partial}_{M} u^{j} \cdot d(\zeta-z)}{u^{j} \cdot(\zeta-z)}\right]^{k}
$$

on $V-A_{j}$.

Proof. The proof is clear after noting that

$$
\begin{aligned}
\bar{\partial}_{M} \alpha_{j}= & {\left[\frac{\bar{\partial}_{M} u^{j} \cdot d(\zeta-z)}{u^{j}(\zeta, z) \cdot(\zeta-z)}\right]_{T_{M \times M}} } \\
& -\left[\frac{\bar{\partial}_{M}\left\{u^{j} \cdot(\zeta-z)\right\} \wedge u^{j} \cdot d(\zeta-z)}{\left(u^{j}(\zeta, z) \cdot(\zeta-z)\right)^{2}}\right]_{T_{M \times M}} \cdot \quad \text { Q.E.D. }
\end{aligned}
$$

We shall also use the following multi-index notation:

If $\beta=\left(\beta_{1}, \ldots, \beta_{N}\right)$ is an $N$-tuple of nonnegative integers, then we let

$$
|\beta|=\beta_{1}+\cdots+\beta_{N} ; \quad\left(\bar{\partial}_{M} \alpha\right)^{\beta}=\left(\bar{\partial}_{M} \alpha_{1}\right)^{\beta_{1}} \wedge \cdots \wedge\left(\bar{\partial}_{M} \alpha_{N}\right)^{\beta_{N}} .
$$

If $I=\left\{i_{1}, \ldots, i_{p}\right\}$ is a $p$-tuple of positive integers, then we let $\alpha_{I}=\alpha_{i_{1}} \wedge \cdots \wedge \alpha_{i_{p}}$ and

$$
E_{I}\left(u^{i_{1}}, \ldots, u^{i_{p}}\right)=(2 \pi i)^{-n}\left\{\alpha_{I} \wedge \sum_{\substack{|\beta|=n-p \\ \beta=\left(\beta_{1}, \ldots, \beta_{p}\right)}}\left(\bar{\partial}_{M} \alpha\right)^{\beta}\right\}
$$

If the $u^{i}$ are understood, then we just write $E_{I}$ for $E_{I}\left(u^{i_{1}}, \ldots, u^{i_{p}}\right)$. The form $E_{I}$ is a smooth form on $V-\left\{\bigcup_{j=1}^{p} A_{i_{j}}\right\}$.

For $1 \leqslant j \leqslant p$, we set $I_{j}=\left\{i_{1}, \ldots, \widehat{i_{j}}, \ldots, i_{p}\right\}$ where we mean that the element $i_{j}$ is omitted. The formal identity we need from Harvey and Polking [HP, Theorem 4.10] is the following:

4.3. TheOREM. On the set $V-\left\{\cup_{j=1}^{p} A_{i_{j}}\right\}$, we have

$$
\bar{\partial}_{M} E_{I}=\sum_{j=1}^{p}(-1)^{j} E_{i_{j}}
$$

where, as before, $\bar{\partial}_{M}$ is taken in both $\zeta$ and $z$.

In the next section, we will take $p=4$ and construct $u^{1}, u^{2}, u^{3}, u^{4}$ so that the resulting kernels defined above will locally solve the tangential Cauchy-Riemann equations.

5. The local solution to $\bar{\partial}_{S}$ in codimension two. In this section, we gather together the notation and identities from $\$ 4$ and the geometric results from $\$ 3$ and prove our local solvability result. 
5.1. TheOREM. Suppose $S$ is a real analytic regular submanifold of $\mathbf{C}^{n}$ of real dimension $2 n-2$. Suppose ex $\operatorname{dim}(S)=1$ and that $S$ is strictly pseudoconvex at a point $x_{0} \in S$. Then there exists a local neighborhood basis of open sets for $S,\left\{\omega_{\alpha}\right\}$, about $x_{0}$ such that the following holds: For each $\omega_{\alpha}$, there is an operator $K: \Gamma_{S}^{p, q}\left(\bar{\omega}_{\alpha}\right) \rightarrow$ $\Gamma_{S}^{p, q-1}\left(\omega_{\alpha}\right)$ such that if $f \in \Gamma_{S}^{p, q}\left(\bar{\omega}_{\alpha}\right), 1 \leqslant q \leqslant n-3$, and $\bar{\partial}_{S} f=0$ on $\omega_{\alpha}$, then $\bar{\partial}_{S}\{K(f)\}=$ fon $\omega_{\alpha}$.

This theorem provides a Poincare lemma for $\bar{\partial}_{S}$ and, as will be seen from the proof, an explicit way of calculating the solution with kernels. The assumption that $f \in \Gamma_{S}^{p, q}\left(\bar{\omega}_{\alpha}\right)$ means that $f$ is smooth on $\bar{\omega}_{\alpha}$.

Most of the rest of this section will be devoted to the proof of this theorem.

Using Theorem 3.8, we may find hypersurfaces $M=\left\{z \in \mathbf{C}^{n} ; \rho(z)=0\right\}$ and $N=\left\{z \in \mathbf{C}^{n} ; r(z)=0\right\}$ with $S=M \cap N$. We may assume $\rho$ is strictly plurisubharmonic near $x_{0}$ and $\partial \bar{\partial} r=0$ near $x_{0}$ in $C^{n}$. By a local biholomorphic change of variables (see Hörmander [Ho, proof of Theorem 2.6.13]) we may assume that $x_{0}=0$ and that $M \cap U$ is a strictly convex hypersurface in $U$, where $U$ is some open ball on $\mathbf{C}^{n}$ containing $x_{0}=0$. In fact, we may choose local holomorphic coordinates so that

$$
\rho(z)=\operatorname{Re} z_{1}-v\left(\operatorname{Im} z_{1}, z_{2}, \ldots, z_{n}\right)
$$

where $v$ is a strictly convex function near the origin with $v(0)=(\nabla v)(0)=0$. $(v$ is strictly convex near the origin if and only if the real Hessian of $v$ at the origin is positive definite.) By possibly shrinking $U$, we may find a holomorphic function $h$ on $U$ with $\operatorname{Re} h=r$ on $U$.

Let $W_{\alpha}=U \cap M \cap\left\{z \in \mathbf{C}^{n} ; \operatorname{Re} z_{1}<\alpha\right\}$. Since $v$ is a strictly convex function, it is clear that the collection of open sets $\left\{W_{\alpha}, \alpha>0\right\}$ forms a local neighborhood basis for $M$ about the point $x_{0}=0$. Therefore, the collection $\left\{\omega_{\alpha}=W_{\alpha} \cap S, \alpha>0\right\}$ forms a local neighborhood basis of open sets for $S$ about the point $x_{0}=0$.

From now on we fix $\alpha>0$ small enough so that $W_{\alpha} \subset \subset U$.

We now wish to construct functions $u^{1}, u^{2}, u^{3}, u^{4}: U \times U \rightarrow \mathrm{C}^{n}$ so that the resulting kernels constructed in $\$ 4$ can be used in the proof of Theorem 5.1. We let

$$
\begin{aligned}
u^{1}(\zeta, z) & =\left(\partial \rho(\zeta) / \partial \zeta_{1}, \ldots, \partial \rho(\zeta) / \partial \zeta_{n}\right), \\
u^{2}(\zeta, z) & =\left(\partial \rho(z) / \partial z_{1}, \ldots, \partial \rho(z) / \partial z_{n}\right)
\end{aligned}
$$

So $u^{1}$ is just the complex gradient of $\rho$ at $\zeta$, and we will think of $u^{1}$ and $u^{2}$ as depending on both variables $(\zeta, z)$.

An easy power series argument shows that there exists a holomorphic mapping $v$ : $U \times U \rightarrow \mathbf{C}^{n}, v=\left(v_{1}, \ldots, v_{n}\right)$, such that

$$
h(\zeta)-h(z)=\sum_{j=1}^{n} v_{j}(\zeta, z)\left(\zeta_{j}-z_{j}\right)=v(\zeta, z) \cdot(\zeta-z)
$$

for $(\zeta, z) \in U \times U$. We shall let $u^{3}=v$. Finally, we shall let $u^{4}(\zeta, z)$ be the constant vector $(1,0, \ldots, 0)$.

We shall need the following kernels constructed in $\S 4: E_{123}, E_{13}, E_{23}, E_{1234}, E_{134}$, $E_{234}, E_{124}$. Using Proposition 4.2, and the fact that $\bar{\partial} u^{3}=\bar{\partial} u^{4}=0$, we can write out 
these kernels. For example:

$$
\begin{array}{r}
E_{134}(\zeta, z)=(2 \pi i)^{-n}\left\{\left[\frac{\partial \rho(\zeta) / \partial \zeta \cdot \dot{d}(\zeta-z)}{\partial \rho(\zeta) / \partial \zeta \cdot(\zeta-z)}\right] \wedge\left[\frac{u^{3}(\zeta, z) \cdot d(\zeta-z)}{u^{3}(\zeta, z) \cdot(\zeta-z)}\right]\right. \\
\left.\wedge \frac{d\left(\zeta_{1}-z_{1}\right)}{\left(\zeta_{1}-z_{1}\right)} \wedge\left[\frac{\bar{\partial}_{\zeta} u^{1} \cdot d(\zeta-z)}{u^{1}(\zeta, z) \cdot(\zeta-z)}\right]^{n-3}\right\}_{T_{M \times M}},
\end{array}
$$

$E_{1234}(\zeta, z)=(2 \pi i)^{-n}\left\{\left(\frac{u^{1} \cdot d(\zeta-z)}{u^{1} \cdot(\zeta-z)}\right) \wedge\left(\frac{u^{2} \cdot d(\zeta-z)}{u^{2} \cdot(\zeta-z)}\right)\right.$

$$
\begin{aligned}
& \wedge\left(\frac{u^{3} \cdot d(\zeta-z)}{u^{3} \cdot(\zeta-z)}\right) \wedge\left(\frac{d\left(\zeta_{1}-z_{1}\right)}{\left(\zeta_{1}-z_{1}\right)}\right) \\
& \wedge \sum_{j+k=n-4}\left(\frac{\bar{\partial}_{\zeta} u^{1} \cdot d(\zeta-z)}{u^{1} \cdot(\zeta-z)}\right)^{j} \\
&\left.\wedge\left(\frac{\bar{\partial}_{z} u^{2} \cdot d(\zeta-z)}{u^{2} \cdot(\zeta-z)}\right)^{k}\right\}_{T_{M \times M}}
\end{aligned}
$$

Let $V=\{M \times M\} \cap\{U \times U\}$. We have the following equation from Theorem 4.3:

$$
\bar{\partial}_{M} E_{1234}=E_{123}-E_{124}+E_{134}-E_{234}
$$

which holds on $V-\left\{\cup_{i=1}^{4} A_{i}\right\}$, where

$$
A_{i}=\left\{(\zeta, z) \in V ; u^{i}(\zeta, z) \cdot(\zeta-z)=0\right\}
$$

Let us carefully analyze $A_{1}, A_{2}, A_{3}, A_{4}$. Since $M \cap U$ is strictly convex, $u^{1}$ is a strong support function for $M \cap U$, as defined in [AB, Definition 3.4]. In particular, this means that $u^{1}(\zeta, z)$ is holomorphic in $z\left(u^{1}\right.$ is, in fact, independent of $\left.z\right)$ and that

$$
A_{1}=\{(\zeta, z) \in V ; \partial \rho(\zeta) / \partial \zeta \cdot(\zeta-z)=0\}=\{(z, z) \in V\} \text {. }
$$

That is, $A_{1}$ is just the diagonal of $V$. Indeed, if $\zeta \in M$ and $\partial \rho(\zeta) / \partial \zeta \cdot(\zeta-z)=0$, then $(\zeta-z)$ must be a holomorphic tangent vector of $M$ at $\zeta$; and the strict convexity of $M \cap U$ implies that $z \notin M$; unless $z=\zeta$. Similarly,

$$
A_{2}=\{(\zeta, z) \in V ; \partial \rho(z) / \partial z \cdot(\zeta-z)=0\}=\{(z, z) \in V\}=A_{1} .
$$

Now, $A_{3}=\{(\zeta, z) \in V ; h(\zeta)=h(z)\}$ and $A_{4}=\left\{(\zeta, z) \in V ; \zeta_{1}=z_{1}\right\}$. Clearly, both $A_{1}$ and $A_{2}$ are contained in $A_{3} \cap A_{4}$.

Let $E$ denote any one of the kernels $E_{123}, E_{13}, E_{23}$. If $z \in U \cap M$ is fixed, then the $\zeta$-singular support of $E(\zeta, z)$ on $M \cap U$ is contained in the set $A_{3}^{z}=\{\zeta \in M \cap U$; $h(\zeta)=h(z)\}$. For $|t|$ small, let $S_{t}=\{\zeta \in M \cap U ; r(\zeta)=t\}$. If $r(z)=t$, then it is clear that $A_{3}^{z} \subset S_{t}$ because $r=\operatorname{Re} h$. Therefore, if $f \in \mathscr{D}_{S}^{p, q}(S \cap U)$, then

$$
E\left([S]^{0,2} \wedge f\right)(z)=\int_{\zeta \in S} E(\zeta, z) \wedge f(\zeta)
$$


defines a smooth form for $z \in\left\{M^{+} \cap U\right\} \cup\left\{M^{-} \cap U\right\}$, where $M^{+}=\{z \in M$; $r(z)>0\}$ and $M^{-}=\{z \in M ; r(z)<0\}$. We shall need the following boundary value result (see Theorem 5.3 in [AB]).

5.5. LeMma. Let $E$ be any one of the kernels $E_{123}, E_{13}, E_{23}$. Suppose $f \in \mathbb{D}_{S}^{*}(S \cap U)$. Suppose $N$ is any nonnegative integer and let $X_{1} \cdots X_{N}$ be vector fields on $M$ with $X_{i}(r)=0$ for $1 \leqslant i \leqslant N\left(\right.$ so $\left.X_{i}\right|_{S}$ is a tangential vector field to $S$ ). Then $X_{1} \cdots$ $\left.X_{n}\left\{E\left([S]^{0,2} \wedge f\right)\right\}\right|_{M^{ \pm} \cap U}$ has a continuous extension to $S \cap U$.

The content of Lemma 5.5 is that the boundary values, from either $\mathrm{M}^{+}$or $\mathrm{M}^{-}$, of any tangential derivative of $E\left([S]^{0,2} \wedge f\right)$ exist on $S \cap U$. Certain stronger boundary value results are true; see [AB] for more details. We shall let $E^{ \pm}(f)$ be the extension of $\left.\left\{E\left([S]^{0,2} \wedge f\right)\right\}_{T_{S}}\right|_{M^{ \pm} \cap U}$ to $S \cap U$. Lemma 5.5 implies that $E^{ \pm}(f) \in \Gamma_{S}^{*}(S \cap U)$, i.e. $E^{ \pm}(f)$ is a smooth tangential form on $S$.

Now we analyse the boundary behavior of $E\left([S]^{0,2} \wedge g\right)$ where $g \in \mathscr{D}_{S}^{*}(S \cap U)$ and $E$ is any one of the kernels $E_{1234}, E_{124}, E_{134}, E_{234}$. A typical coefficient of $E(\zeta, z) \wedge g(\zeta)$ is

$$
g(\zeta) /\left(u^{1} \cdot(\zeta-z)\right)^{p}\left(u^{2} \cdot(\zeta-z)\right)^{q}\left(u^{3} \cdot(\zeta-z)\right)^{l}\left(\zeta_{1}-z_{1}\right)^{k}
$$

where $p, q, l, k$ are nonnegative integers. In general, $E\left([S]^{0,2} \wedge g\right)(z)$ is not even defined for $z \in\left\{M^{+} \cap U\right\} \cap\left\{M^{-} \cap U\right\}$ because of the term $\left(\zeta_{1}-z_{1}\right)^{-k}$. However, if $g \equiv 0$ on $\omega_{\alpha}$ then the term

$$
g(\zeta) /\left(u^{1} \cdot(\zeta-z)\right)^{p}\left(u^{2} \cdot(\zeta-z)\right)^{q}\left(\zeta_{1}-z_{1}\right)^{k}
$$

is smooth for $\zeta \in S$ and $z \in W_{\alpha}$. This is because the numerator is nonzero only when $\operatorname{Re} \zeta_{1}>\alpha$, and the denominators are nonvanishing when $\operatorname{Re} z_{1}<\alpha, \operatorname{Re} \zeta_{1}>\alpha$. Therefore, if $g \equiv 0$ on $\omega_{\alpha}$, then $E\left([S]^{0,2} \wedge g\right)$ is smooth on $W_{\alpha}-S$. Moreover, since $g(\zeta) /\left(\zeta_{1}-z_{1}\right)^{k}$ is smooth for $\zeta \in S$ and $z \in W_{\alpha}$, the boundary behavior of $E\left([S]^{0,2} \wedge g\right)$ on $S \cap W_{\alpha}=\omega_{\alpha}$ is the same (in fact better) than the boundary behavior of $E_{123}\left([S]^{0,2} \wedge \mathrm{g}\right)$. That is, the same proof in [AB] for Lemma 5.5 is valid for the following lemma.

5.5'. Lemma. Let $E$ be any one of the kernels $E_{1234}, E_{134}, E_{124}, E_{234}$. Suppose $g \in \mathscr{D}_{S}^{*}(S \cap U)$ with $g \equiv 0$ on $\omega_{\alpha}$. Suppose $N$ is any nonnegative integer and let $X_{1} \cdots X_{N}$ be vector fields on $M$ with $X_{i}(r)=0$ for $1 \leqslant i \leqslant N$. Then $X_{1}$ $\left.\cdots X_{N}\left\{E\left([S]^{0,2} \wedge g\right)\right\}\right|_{M^{ \pm} \cap W_{\alpha}}$ has a continuous extension to $S \cap W_{\alpha}=\omega_{\alpha}$. to $\omega_{\alpha}$.

Again, we shall let $E^{ \pm}(g) \in \Gamma_{S}^{*}\left(\omega_{\alpha}\right)$ be the extension of $\left.\left\{E\left([S]^{0,2} \wedge g\right)\right\}_{T_{S}}\right|_{M^{ \pm} \cap W_{\alpha}}$

As the next lemma shows, many of the kernels defined above act nontrivially only on forms having a particular bidegree.

5.7. Lemma. (a) Suppose $f \in \mathscr{D}_{S}^{p, q}(S \cap U)$; then:

$$
\begin{array}{ll}
E_{13}\left([S]^{0,2} \wedge f\right)=0 & \text { unless } q=0, \\
E_{23}\left([S]^{0,2} \wedge f\right)=0 & \text { unless } q=n-2 .
\end{array}
$$


(b) Suppose $g \in \mathscr{D}_{S}^{p, q}(S \cap U)$ and $g \equiv 0$ on $\omega_{\alpha}$; then:

$$
\begin{array}{ll}
E_{134}\left([S]^{0,2} \wedge g\right)=0 & \text { unless } q=1, \\
E_{234}\left([S]^{0,2} \wedge g\right)=0 & \text { unless } q=n-2
\end{array}
$$

Proof. For example, the kernel $E_{134}(\zeta, z)$ is written out in (5.2). Since $u^{1}$ does not depend on $z, E_{134}(\zeta, z)$ is of degree $n-3$ in $d \bar{\zeta}$. Therefore, on $S, E_{134}(\zeta, z)$ will only pair with the piece of $g(\zeta)$ of type $(p, 1)$ (to add up to $n-2 d \bar{\zeta}$ 's). Similarly, the degree of $E_{13}, E_{23}$ and $E_{234}$ in $d \bar{\zeta}$ are, respectively, $n-2$, zero, and zero. Q.E.D.

We must put the kernel $E_{124}$ in a special category. Formally, $E_{124}^{\prime}$ acts nontrivially on all bidegrees. However, soon we will be interested in the boundary value jump of these kernels across $S$. If $g \equiv 0$ on $\omega_{\alpha}$, then we claim that the boundary value jump of $E_{124}\left([S]^{0,2} \wedge g\right)$ across $S \cap W_{\alpha}=\omega_{\alpha}$ vanishes, i.e.,

$$
\left(E_{124}^{+}-E_{124}^{-}\right)(g)=0 \text { on } \omega_{\alpha} \text {. }
$$

To see this, note that a typical term of $E_{124}(\zeta, z) \wedge g(\zeta)$, given in (5.6), is smooth for $\zeta \in S$ and $z \in W_{\alpha}$. This is because the term $u^{3}(\zeta, z) \cdot(\zeta-z)$ is missing from the denominator. Therefore, if $g \equiv 0$ on $\omega_{\alpha}$, then $E_{124}\left([S]^{0,2} \wedge g\right)$ is smooth on $W_{\alpha}$ (whereas, if $E$ is any of the other kernels, then $E\left([S]^{0,2} \wedge g\right)$ is only a priori smooth on $W_{\alpha}-S$ ). Of course, the boundary value jump across $S \cap W_{\alpha}$ of a smooth form on $W_{\alpha}$ is zero.

We now summarize the "global" solution to $\bar{\partial}_{S}$ given in $[\mathbf{A B}]$. let

$$
\begin{aligned}
& E=E_{123}^{+}-E_{123}^{-}: \mathscr{D}_{S}^{p, q}(S \cap U) \rightarrow \Gamma_{S}^{p, q-1}(S \cap U), \\
& Q=\left(E_{13}^{+}-E_{13}^{-}\right)+\left(E_{23}^{-}-E_{23}^{+}\right): \mathscr{D}_{S}^{p, q}(S \cap U) \rightarrow \Gamma_{S}^{p, q}(S \cup U) .
\end{aligned}
$$

From formula (5.9) in $\$ 5$ of [AB], we have

$$
I=Q+\bar{\partial}_{S} \circ E+E \circ \bar{\partial}_{S} \quad(I=\text { identity }) .
$$

This holds as an operator equation from $\mathscr{D}_{S}^{p, q}(S \cap U)$ to $\Gamma_{\xi}^{p, q}(S \cap U)$. Actually, the operators $E$ and $Q$ constructed in $[\mathbf{A B}]$ act on the space $\mathscr{D}_{S}^{p, q}(S)$. But since $h$ is only defined on $U$, the kernels $E_{123}, E_{13}$ and $E_{23}$ are only defined on $U \times U$. Hence these kernels only act on $\mathscr{Q}_{S}^{p, q}(S \cap U)$.

If $f \in \mathscr{Q}_{S}^{p, q}(S \cap U)$ with $1 \leqslant q \leqslant n-3$, then $Q(f)=0$ by Lemma 5.7, and we have

$$
f=\bar{\partial}_{S}\{E(f)\}+E\left(\bar{\partial}_{S} f\right) \text { on } S \cap U \text {. }
$$

Note that this equation does not solve our local problem in Theorem 5.1, because we only assume $\bar{\partial}_{S} f=0$ on $\omega_{\alpha}$ (and not on all of $S \cap U$ ). However, we are now in a position to complete the proof of Theorem 5.1. Suppose first that $f \in \Gamma_{S}^{p, q}\left(\bar{\omega}_{\alpha}\right)$, $1 \leqslant q \leqslant n-4$, and satisfies $\bar{\partial}_{S} f=0$ on $\omega_{\alpha}$; let $\tilde{f} \in \mathcal{D}_{S}^{p, q}(S \cap U)$ be a smooth extension of $f$. Clearly, $\operatorname{supp}\left\{\bar{\partial}_{M} \tilde{f}\right\} \subset U \cap\left\{S-\omega_{\alpha}\right\}$. Using (5.10), we have

$$
f=\tilde{f}=\bar{\partial}_{S}\{E(\tilde{f})\}+E\left(\bar{\partial}_{S} \tilde{f}\right) \text { on } \omega_{\alpha} \text {. }
$$


Using (5.4) we have

$$
\begin{aligned}
E_{123}\left([S]^{0,2} \wedge \bar{\partial}_{S} \tilde{f}\right)= & \bar{\partial}_{M}\left\{E_{1234}\left([S]^{0,2} \wedge \bar{\partial}_{S} \tilde{f}\right)\right\} \\
& +\left(E_{124}-E_{134}+E_{234}\right)\left([S]^{0,2} \wedge \bar{\partial}_{S} \tilde{f}\right) .
\end{aligned}
$$

This equation makes sense on $W_{\alpha}-S$ since $\bar{\partial}_{S} \tilde{f}=0$ on $\omega_{\alpha}$. We have also used equation (4.1) and the fact that $\bar{\partial}_{S} \bar{\partial}_{S} \tilde{f}=0$. By Lemma 5.7,

$$
E_{134}\left([S]^{0,2} \wedge \bar{\partial}_{S} \tilde{f}\right)=E_{234}\left([S]^{0,2} \wedge \bar{\partial}_{S} \tilde{f}\right)=0 .
$$

So,

$$
E_{123}\left([S]^{0,2} \wedge \bar{\partial}_{S} \tilde{f}\right)=\bar{\partial}_{M}\left\{E_{1234}\left([S]^{0,2} \wedge \bar{\partial}_{S} \tilde{f}\right)\right\}+E_{124}\left([S]^{0,2} \wedge \bar{\partial}_{S} \tilde{f}\right)
$$

As before, we let $S_{t}=\{\zeta \in M \cap U ; r(\zeta)=t\}$. If we apply the projection map $T_{S}$ to equation (5.13) and then restrict this equation to $S_{t}, t \neq 0$, we obtain

$$
\left\{E_{123}\left([S]^{0,2} \wedge \bar{\partial}_{S} \tilde{f}\right)\right\}_{T_{S}}=\bar{\partial}_{S_{t}}\left\{E_{1234}\left([S]^{0,2} \wedge \bar{\partial}_{S} \tilde{f}\right)\right\}+\left\{E_{124}\left([S]^{0,2} \wedge \bar{\partial}_{S} \tilde{f}\right)\right\}_{T_{S}}
$$

on $S_{t} \cap W_{\alpha}$. We have used the fact that if $F$ is a smooth form, then $\left\{\bar{\partial}_{M} F\right\}_{T_{S}}=$ $\bar{\partial}_{S_{t}}\left\{F_{T_{s}}\right\}$ on $S_{t}$ (see $\left.\S_{4}\right)$. Now $\bar{\partial}_{S_{t}}$ involves vector fields $X$ which annihilate $r(X(r)=0)$. Therefore, by Lemmas 5.5 and $5.5^{\prime}$, we can take boundary values of the above equation from $M^{+} \cap W_{\alpha}$ or $M^{-} \cap W_{\alpha}$ (i.e., let $t \rightarrow 0^{+}$or $t \rightarrow 0^{-}$). We obtain

$$
E_{123}^{ \pm}\left(\bar{\partial}_{S} \tilde{f}\right)=\bar{\partial}_{S}\left\{E_{1234}^{ \pm}\left(\bar{\partial}_{S} \tilde{f}\right)\right\}+E_{124}^{ \pm}\left(\bar{\partial}_{S} \tilde{f}\right) \text { on } \omega_{\alpha} \text {. }
$$

Set $\varepsilon=E_{1234}^{+}-E_{1234}^{-}$. By Lemma $5.5^{\prime}, \mathcal{E}\left(\bar{\partial}_{S} \tilde{f}\right) \in \Gamma_{S}^{p, q-1}\left(\omega_{\alpha}\right)$. If we subtract $E_{123}^{-}\left(\bar{\partial}_{S} \tilde{f}\right)$ from $E_{123}^{+}\left(\bar{\partial}_{S} \tilde{f}\right)$ on $\omega_{\alpha}$ and use (5.8), we obtain

$$
E\left(\bar{\partial}_{S} \tilde{f}\right)=\left(E_{123}^{+}-E_{123}^{-}\right)\left(\bar{\partial}_{S} \tilde{f}\right)=\bar{\partial}_{S}\left\{\mathcal{E}\left(\bar{\partial}_{S} \tilde{f}\right)\right\} \quad \text { on } \omega_{\alpha} \text {. }
$$

Putting (5.10) and (5.14) together, we finally have

$$
f=\bar{\partial}_{S}\left\{E(\tilde{f})+\mathcal{E}\left(\bar{\partial}_{S} \tilde{f}\right)\right\} \quad \text { on } \omega_{\alpha}
$$

as desired. This completes the proof of Theorem 5.1 when $f \in \Gamma_{S}^{p, q}$ and $q \leqslant n-4$.

When $q=n-3$, the above argument is valid except that we may not use Lemma 5.7 to show that $E_{234}\left([S]^{0,2} \wedge \bar{\partial}_{S} \tilde{f}\right)=0$. Instead, we use the following approximation argument used by Henkin in the hypersurface case (cf. [H, p. 90]).

Now clearly

$$
E_{234}\left([S]^{0,2} \wedge \bar{\partial}_{S} \tilde{f}\right)(z)=\int_{\zeta \in S} K(\zeta, z) \wedge \bar{\partial}_{S} f(\zeta)
$$

where $K(\zeta)$ is a $(n-p, 0)$ form with coefficients which are holomorphic in $\zeta$ provided $\zeta_{1} \neq z_{1}, h(\zeta) \neq h(z)$ and $\partial \rho(z) / \partial z \cdot(\zeta-z) \neq 0$. Choose $\beta>\alpha$ so that $\operatorname{supp} \bar{\partial}_{S} \tilde{f} \subset\left\{\zeta \in S ; \alpha \leqslant \operatorname{Re} \zeta_{1} \leqslant \beta\right\}$. Fix $z \in W_{\alpha}-S$ and set $t=r(z) \neq 0$. Let

$$
B_{\varepsilon}=\left\{\zeta \in U ; \rho(z)>-\varepsilon, \alpha-\varepsilon<\operatorname{Re} \zeta_{1}<\beta+\varepsilon \text {, and }-|t| / 2<r(\zeta)<|t| / 2\right\} \text {. }
$$

We can choose $\varepsilon>0$ (depending on $z$ ) so that $K(\zeta, z)$ is holomorphic for $\zeta \in B$. Now recall that $\rho(\zeta)=\operatorname{Re} \zeta_{1}-v\left(\operatorname{Im} \zeta_{1}, \zeta_{2}, \ldots, \zeta_{n}\right)$. (with $v$ strictly convex on $U$ ) and

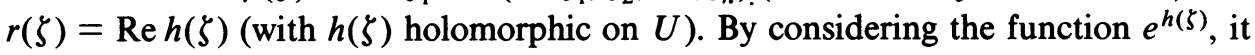
is easy to see that $B_{\varepsilon}$ is relatively Runge in the open ball $U$. Therefore there is a 
sequence $\left\{K_{j}(\zeta)\right\}$ of holomorphic $(n-p, 0)$ forms on $U$ which converge uniformly on $\left\{\zeta \in S, \alpha \leqslant \operatorname{Re} \zeta_{1} \leqslant \beta\right\} \subset \subset B$ to $K(\zeta, z)$. Thus,

$$
\begin{aligned}
E_{234}\left([S]^{0,2} \wedge \bar{\partial}_{S} f\right)(z) & =\lim _{j \rightarrow \infty} \int_{\zeta \in S} K_{j}(\zeta) \wedge \bar{\partial}_{S} f(\zeta) \\
& =(-1)^{n-p+1} \lim _{j \rightarrow \infty} \int_{z \in S} \bar{\partial}_{S} K_{j}(\zeta) \wedge f(\zeta)=0
\end{aligned}
$$

as desired. This completes the proof of Theorem 5.1. Q.E.D.

REMARKS. (1) The solution, given in (5.15) to $\bar{\partial}_{S} u=f$, is valid for any smooth extension $\tilde{f}$ with compact support in $U \cap S$. It is suggestive to try a particular nonsmooth extension, $\tilde{f}=\chi_{\omega_{\alpha}} f$, where $\chi_{\omega_{\alpha}}$ is the characteristic function on the set $\omega_{\alpha}$. Then $\bar{\partial}_{s} \tilde{f}=\left[\partial \omega_{\alpha}\right]^{0,3} \wedge f$ and the solution to $\bar{\partial}_{s} u=f$ on $\omega_{\alpha}$ becomes

$$
u=E\left(\chi_{\omega_{\alpha}} f\right)+\mathcal{E}\left(\left[\partial \omega_{\alpha}\right]^{0,3} \wedge f\right) \text {. }
$$

The above solution only depends on the values of $f$ on $\bar{\chi}_{\omega_{\alpha}}$ (and not on derivatives of $f$ ). This solution may be useful for certain $L^{p}$ and continuity estimates. However, one must make sense out of the terms $E\left(\chi_{\omega_{\alpha}} \wedge f\right)$ and $\mathcal{E}\left(\left[\partial \omega_{\alpha}\right]^{0,3} \wedge f\right)$ as smooth (or continuous) forms on $\omega_{\alpha}$. This, of course, is not implied by the boundary value theorems in $[\mathbf{A B}]$ which we used for the proofs of Lemmas 5.5 and 5.5'. We feel certain, however, that such an analysis can be carried through using similar techniques as in $[\mathbf{A B}]$.

(2) Looking back at equations (5.12) and (5.13), we see that the kernels $E_{124}, E_{134}$ and $E_{234}$ are the obstructions to the local solution to $\bar{\partial}_{S}$. The kernels $E_{134}$ and $E_{234}$, however, only provide obstructions when they act on $(p, q)$ forms with $q=0$ or $n-2$, by Lemma 5.7. (The kernel $E_{124}$ is not an obstruction for an entirely different reason.) This is where the geometry on $S$ (i.e., ex $\operatorname{dim}(S)=1$ ) really plays an important role. The geometric assumptions on $S$ lead to the construction of $u^{3}$ and $u^{4}$, which are holomorphic in both variables, which in turn is the key fact used in the proof of Lemma 5.7. If the geometry on $S$ were different, then the kernels $E_{134}$ and $E_{234}$ (defined in any way that we can conceive of) would provide very nontrivial obstructions to solving $\bar{\partial}_{S}$.

(3) Looking over the work in $[\mathbf{A B}]$ and in the proof of Theorem 5.1, we see an inductive procedure forming to solve the tangential Cauchy-Riemann equations on higher codimension submanifolds. First, let us review what we have done. Let $M$ be a strictly pseudoconvex hypersurface. Let $S \subset M$ be a submanifold defined by $S=\{z \in M ; \operatorname{Re} h(z)=0\}$ where $h$ is a holomorphic function. In [AB], the fundamental solution to $\bar{\partial}_{S}$ (the operator $E$ ) was derived from the fundamental solution to $\bar{\partial}_{M}$ on $M$. The content of Theorem 5.1 is that this fundamental solution can be modified so that we can locally solve the tangential Cauchy-Riemann equations on $S$.

Now suppose $Q \subset S$ is a generic submanifold defined by $Q=\{x \in S ; \operatorname{Re} k(x)=$ 0 ) where $k$ is a holomorphic function on some open set in $\mathbf{C}^{n}$. Following the same procedure as in $[\mathbf{A B}]$, the fundamental solution for $\bar{\partial}_{Q}$ can be derived from the fundamental solution for $\bar{\partial}_{S}$ on $S$. Then, using the same techniques as those in the 
proof of Theorem 5.1, the fundamental solution to $\bar{\partial}_{Q}$ can be modified so that we can locally solve $\bar{\partial}_{Q}$, etc., etc. So, with the help of Theorem 3.4 , one can prove the following analogue of Theorem 5.1 for higher codimension.

5.1'. TheOREM. Suppose $S$ is a real analytic regular submanifold of $\mathbf{C}^{n}$ of real dimension $2 n-k$. Suppose ex $\operatorname{dim}(S)=1$ and that $S$ is strictly pseudoconvex at a point $x_{0} \in S$. Then there exists a local neighborhood basis of open sets $\left\{\omega_{\alpha}\right\}, \alpha>0$, about $x_{0}$ such that the following holds: For each $\alpha>0$, there is an operator $K$ : $\Gamma_{S}^{p, q}\left(\overline{\omega_{\alpha}}\right) \rightarrow \Gamma_{S}^{p, q-1}\left(\omega_{\alpha}\right)$ such that if $f \in \Gamma_{S}^{p, q-1}\left(\overline{\omega_{\alpha}}\right), 1 \leqslant q \leqslant n-k-1$, and $\bar{\partial}_{S} f=0$ on $\bar{\omega}_{\alpha}$, then $\bar{\partial}_{S}\{K(f)\}=f$ on $\omega_{\alpha}$.

We feel no need to publish the proof of Theorem 5.1', because the details would be too repetitious of those in $[\mathbf{A B}]$ and in the proof of Theorem 5.1. Instead, we would like to raise the following interesting questions:

(1) Can we remove the real analytic assumption in Theorem 5.1 (and Theorem $\left.5.1^{\prime}\right)$ ?

The trick here would be to come up with a correct replacement for the function $u^{3}$ used in the proof of Theorem 5.1.

(2) For what class of submanifolds $S$ with ex $\operatorname{dim}(S)>1$ can we locally solve the tangential Cauchy-Riemann equations with kernels?

We feel that there is some suitable answer to question (2), however the bidegree range in which we could solve the tangential Cauchy-Riemann equations would probably be more restrictive than the bidegree range handled in Theorem 5.1.

In Theorem 5.1, we were only interested in forms $f \in \Gamma_{S}^{p, q}\left(\overline{\omega_{\alpha}}\right)$ where $1 \leqslant q \leqslant n-$ 3. However, some interesting information can be obtained when $q=0$. Our next theorem is our boundary value jump result for CR-functions. (A CR-function $f$ on $\omega_{\alpha} \subset S$ satisfies $\bar{\partial}_{S} f=0$ on $\omega_{\alpha}$; see the definition of $\bar{\partial}_{S}$ in $\S 4$ and the remark after Definition 3.2.)

For $V$ an open set in $\mathbf{C}^{n}$, let $\Theta(V)$ denote the space of holomorphic functions on $V$.

5.15'. Theorem. Let $S$ and $x_{0} \in S$ satisfy the hypothesis of Theorem 5.1. Locally there exists a real hypersurface $M$ such that $S \subset M$ and $S$ divides $M$ into two pieces $M^{+}$and $M^{-}$, and there exists a local neighborhood basis $\left\{W_{\alpha}\right\}$ for $M$ about the point $x_{0}$ such that the following holds: For each $W_{\alpha}$ there exist open sets $V_{\alpha}^{-}$and $V_{\alpha}^{+}$in $\mathbf{C}^{n}$ which contain $W_{\alpha} \cap M^{-}$and $W_{\alpha} \cap M^{+}$, respectively, such that $V_{\alpha}^{+} \cap V_{\alpha}^{-}=\varnothing$, $W_{\alpha} \cap S \subset \overline{V_{\alpha}^{+}} \cap \frac{\alpha}{V_{\alpha}^{-}}$, and, moreover, given $f \in \Gamma^{p, o}\left(\overline{W_{\alpha}} \cap S\right)$ with $\overline{\partial_{S}} f=0$ on $\overline{W_{\alpha}} \cap S$, there exists $F \in \theta\left(V_{\alpha}^{+} \cup V_{\alpha}^{-}\right)$such that $\left.F\right|_{M^{-} \cap W_{\alpha}}$ and $\left.F\right|_{M^{+} \cap W_{\alpha}}$ have continuous extensions to $W_{\alpha} \cap S$, denoted by $F^{-}$and $F^{+}$, respectively; and, furthermore, $f=F^{+}$ $-F^{-}$on $W_{\alpha} \cap S$.

The theorem roughly states that a CR- $(p, o)$ form on $\bar{W}_{\alpha} \cap S$ is the boundary value jump of a holomorphic $(p, o)$ form defined on an open set, which only depends on $W_{\alpha} \cap S$.

Proof of Theorem 5.15'. Let $M, M^{+}, M^{-}, W_{\alpha}, \omega_{\alpha}$, and $U$ be defined as in the proof of Theorem 5.1. Let $\tilde{f} \in \mathcal{D}_{S}^{p, o}(S \cap U)$ be a smooth extension of the given 
form $f$, with $\overline{\partial_{S}} \tilde{f}=0$ on $\overline{\omega_{\alpha}}$. We first note that

$$
E_{123}\left([S]^{0,2} \wedge \tilde{f}\right)(z)=\int_{\zeta \in S} E_{123}(\zeta, z) \wedge \tilde{f}(\zeta)=0
$$

and

$$
E_{1234}\left([S]^{0,2} \wedge \bar{\partial}_{S} \tilde{f}\right)(z)=\int_{\zeta \in S} E_{1234}(\zeta, z) \wedge \bar{\partial}_{S} \tilde{f}(\zeta)=0
$$

because of type considerations. For example, the degree of $E_{123}(\zeta, z) \wedge \tilde{f}(\zeta)$ in $d \bar{\zeta}$ is less than $n-2$.

(5.9) and Lemma 5.7(a), therefore, yield

$$
\tilde{f}=\left(E_{13}^{+}-E_{13}^{-}\right)(\tilde{f})+E\left(\bar{\partial}_{S} \tilde{f}\right) \text { on } \omega_{\alpha} .
$$

(5.12) and Lemma 5.7(b) yield

$$
E_{123}\left([S]^{0,2} \wedge \bar{\partial}_{S} \tilde{f}\right)=\left(E_{124}-E_{134}\right)\left([S]^{0,2} \wedge \bar{\partial}_{S} \tilde{f}\right)
$$

on $W_{\alpha}-S$. Taking the boundary value jump of this equation across $\omega_{\alpha}$, and taking into account (5.8), we obtain

$$
E\left(\bar{\partial}_{S} \tilde{f}\right)=\left(E_{123}^{+}-E_{123}^{-}\right)\left(\bar{\partial}_{S} \tilde{f}\right)=\left(E_{134}^{-}-E_{134}^{+}\right)\left(\bar{\partial}_{S} \tilde{f}\right) .
$$

In view of (5.16) and (5.17), we finally have

$$
f=\left(E_{13}^{+}-E_{13}^{-}\right)(\tilde{f})+\left(E_{134}^{-}-E_{134}^{+}\right)\left(\bar{\partial}_{S} \tilde{f}\right) \text { on } \omega_{\alpha} .
$$

Now, the functions $u^{1}, u^{2}, u^{3}, u^{4}$ are defined on $U \times U \subset \mathbf{C}^{n} \times \mathbf{C}^{n}$. Therefore, we may examine all the kernels defined above on $U \times U$, provided we avoid their singular sets. Define

$$
\begin{aligned}
F(z) & =E_{13}\left([S]^{0,2} \wedge \tilde{f}\right)(z)-E_{134}\left([S]^{0,2} \wedge \bar{\partial}_{S} \tilde{f}\right)(z) \\
& =\int_{\zeta \in S} E_{13}(\zeta, z) \wedge \tilde{f}(\zeta)-\int_{\zeta \in S} E_{134}(\zeta, z) \wedge \bar{\partial}_{S} \tilde{f}(\zeta)
\end{aligned}
$$

and let

$$
\tilde{A}_{i}^{z}=\left\{\zeta \in \bar{U} ; u^{i}(\zeta, z) \cdot(\zeta-z)=0\right\}
$$

Clearly, $F(z)$ is smooth at a point $z \in U$ provided

$$
\tilde{A}_{1}^{z} \cap S=\tilde{A}_{3}^{z} \cap S=\varnothing
$$

and

$$
\tilde{A}_{4}^{z} \cap\left\{\operatorname{supp} \bar{\partial}_{S} \tilde{f}\right\} \cap S=\varnothing .
$$

We have already seen that if $z \in W_{\alpha}-S$ then

$$
\tilde{A}_{1}^{z} \cap S=\tilde{A}_{3}^{z} \cap S=\varnothing .
$$

Since each $\tilde{A}_{i}^{z}$ is compact in $\mathbf{C}^{n}$ for each $z$, it is clear that there is some neighborhood $V_{1}$ in $\mathbf{C}^{n}$ containing $W_{\alpha}-S$ such that (5.21) remains true for $z \in V_{1}$. 
Recall that $u^{4}(\zeta, z) \cdot(\zeta-z)=\zeta_{1}-z_{1}$. Since $\bar{\partial}_{S} \tilde{f}(\zeta)=0$ for $\operatorname{Re} \zeta_{1} \leqslant \alpha,(5.20)$ clearly holds for $z$ with $\operatorname{Re} z_{1}<\alpha$. Therefore if we let

$$
\begin{aligned}
& V_{\alpha}^{+}=V_{1} \cap\left\{z \in U ; \operatorname{Re} z_{1}<\alpha\right\} \cap\{z \in U ; r(z)>0\}, \\
& V_{\alpha}^{-}=V_{1} \cap\left\{z \in U ; \operatorname{Re} z_{1}<\alpha\right\} \cap\{z \in U ; r(z)<0\},
\end{aligned}
$$

then $F$ is smooth on $V_{\alpha}^{+} \cup V_{\alpha}^{-}$. Moreover, $F$ is holomorphic on $V_{\alpha}^{+} \cup V_{\alpha}^{-}$because $u^{1}(\zeta, z), u^{3}(\zeta, z)$, and $u^{4}(\zeta, z)$ are holomorphic functions in $z$ for fixed $\zeta$. Finally, (5.18) implies

$$
f=F^{+}-F^{-} \text {on } \omega_{\alpha} \text {. Q.E.D. }
$$

EXAmple. Suppose $S=\left\{z \in \mathbf{C}^{n} ;|z|=1\right\} \cap\left\{z \in \mathbf{C}^{n} ; \operatorname{Re} z_{n}=0\right\}$ is the equator of the unit sphere in $\mathbf{C}^{n}$. $S$ satisfies the hypothesis of Theorem 5.1. We have $\rho(\zeta)=|\zeta|^{2}-1, u^{1}(\zeta, z)=\bar{\zeta}, u^{2}(\zeta, z)=\bar{z}$, and $u^{3}(\zeta, z)=(0, \ldots, 1)$. Suppose $\omega_{\alpha}=$ $\left\{z \in S ; \operatorname{Re} z_{1}>1-\alpha\right\}$. Then we may take $u^{4}(\zeta, z)=(1,0, \ldots, 0)$. Theorem 5.1 says that we can solve $\bar{\partial}_{s}$ on $\omega_{\alpha}$ on bidegrees $(p, q), 1 \leqslant q \leqslant n-3$. Theorem $5.15^{\prime}$ says that a CR-function on $\omega_{\alpha}$ is the boundary value jump across $\omega_{\alpha}$ of a holomorphic function defined on $V_{\alpha}=V_{\alpha}^{+} \cup V_{\alpha}^{-}$where we may take

$$
\begin{gathered}
V_{\alpha}^{+}=\left\{z \in \mathbf{C}^{n} ; \operatorname{Re} z_{n}>0 ; \operatorname{Re} z_{1}>1-\alpha\right. \text { and } \\
\left.\left|z_{1}\right|^{2}+\cdots+\left|z_{n-1}\right|^{2}+\left(\operatorname{Im} z_{n}\right)^{2}<1\right\}, \\
V_{\alpha}^{-}=\left\{z \in \mathbf{C}^{n} ; \operatorname{Re} z_{n}<0 ; \operatorname{Re} z_{1}>1-\alpha\right. \text { and } \\
\left.\left|z_{1}\right|^{2}+\cdots+\left|z_{n-1}\right|^{2}+\left(\operatorname{Im} z_{n}\right)^{2}<1\right\} .
\end{gathered}
$$

The set $V_{\alpha}$ is constructed to be the largest open set such that (5.19) and (5.20) hold.

It is natural to ask whether or not one can arrange that one of $F^{+}$or $F^{-}$vanish. That is, does there exist an open set $V$ in $\mathbf{C}^{n}$ with $\omega_{\alpha} \subset \bar{V} \cap S$ such that any CR-function on $\omega_{\alpha}$ extends to a holomorphic function on $V$. For example, the answer is affirmative if ex $\operatorname{dim}(S)=\operatorname{codim}_{\mathbf{R}}(S)$; see for example [HW]. As the next theorem shows, however, the answer is negative when $S$ is real analytic and ex $\operatorname{dim}(S)<\operatorname{codim}_{\mathbf{R}}(S)$. Thus, the boundary value jump result in the previous theorem is the best possible result.

5.22. TheOREM. Suppose $S$ is a real analytic, regular submanifold of real dimension $2 n-k$ with ex $\operatorname{dim}(S)<k=\operatorname{codim}_{\mathbf{R}}(S)$. Suppose $x_{0} \in S$ and $\omega$ is any sufficiently small open set in $S$ containing $x_{0}$. There does not exist any open set $V$ in $\mathbf{C}^{n}$ with the following properties:

(1) $\omega \subset \bar{V} \cap S$,

(2) any $C R$-function on $\omega$ has a unique extension to a holomorphic function on $V$.

Proof. Suppose ex $\operatorname{dim}(S)=k-p$ with $p>0$. By Theorem 3.4, there exist hypersurfaces $N_{j}, 1 \leqslant j \leqslant p$, locally defined by $N_{j}=\left\{z \in \mathbf{C}^{n} ; r_{j}(z)=0\right\}$ where each $r_{j}: \mathbf{C}^{n} \rightarrow \mathbf{R}$ is real analytic and $d r_{1} \wedge \cdots \wedge d r_{p} \neq 0$, and there exists an open ball $U$ in $\mathbf{C}^{n}$ containing $x_{0}$ such that $S \cap U \subset N_{1} \cap \cdots \cap N_{p}$ and $\partial \bar{\partial} r_{j}=0$ on $U$. Assume 
that $\omega$ is any open set in $S$ with $\omega \subset U \cap S$. We claim that $U \cap N_{1} \cap \cdots \cap N_{p}$ is the intersection of domains of holomorphy. To this end we write

$$
U \cap N_{1} \cap \cdots \cap N_{p}=\bigcap_{t>0} V_{t}
$$

where

$$
V_{t}=\left\{z \in U ;-t<r_{j}(z)<t, 1 \leqslant j \leqslant p\right\} .
$$

Since $\partial \bar{\partial} r_{j}=0$ on $U$, for $1 \leqslant j \leqslant p$, each $V_{t}$ is clearly a domain of holomorphy. So for each $t>0$, there exists $f_{t} \in \mathcal{O}\left(V_{t}\right)$ such that $f_{t}$ does not analytically continue past any part of $\partial V_{t}$ (see Hörmander [Ho, Theorem 2.55]).

Now suppose, by contradiction, that there exists an open set $V$ in $\mathbf{C}^{n}$ satisfying properties (1) and (2). Choose $t>0$ small enough so that $V \nsubseteq V_{t}$; then $\left.f_{t}\right|_{s}$ is a CR-function on $S \cap U$ which extends to $\tilde{f}_{t} \in \theta(V)$. Since this extension is unique, $f_{t} \in \Theta\left(V_{t}\right)$ has an extension $\tilde{f}_{t} \in \Theta(V)$, which is a contradiction. Q.E.D.

REMARKs. (1) The technique of writing a Levi flat surface, such as $N_{1} \cap \cdots \cap N_{p}$ $\cap U$, as the intersection of domains of holomorphy can also be found in Wells [W].

(2) Theorem 5.22 is not contradictory to Theorem 3.3 of Tomassini (used in the proof of Theorem 3.4) which states that any real analytic CR-function on a real analytic submanifold $S$ is the restriction of a holomorphic function on a neighborhood of $S$. In Tomassini's theorem, the neighborhood is allowed to depend on the CR-function. The content of Theorem 5.22 is that there is no fixed neighborhood to which all CR-functions extend as holomorphic functions.

(3) As mentioned previously in the real analytic case, this result provides a converse to the following result (see Hunt and Wells [HW]): If $S$ is a smooth regular submanifold of $\mathbf{C}^{n}$ with ex $\operatorname{dim}(S)=\operatorname{codim}_{\mathrm{R}}(S)$, then $S$ is locally extendible to an open set in $\mathbf{C}^{n}$. Thus, a real analytic, regular submanifold is extendible if and only if its excess dimension of its Levi algebra is maximal (i.e. ex dim = real codim).

\section{BIBLIOGRAPHY}

[AB] A. Boggess, Kernels for the tangential Cauchy Riemann equations, Ph.D. thesis, Rice Univ., May, 1979; Trans. Amer. Math. Soc. 262 (1980), 1-50.

[C] E. Cartan, Les systèmes différentiels extérieurs et leurs applications géométriques, Hermann, Paris, 1945.

[F] H. Flanders, Differential forms with applications to the physical sciences, Academic Press, New York, 19003.

[G] R. B. Gardner, Invariants of Pfaffian systems, Trans. Amer. Math. Soc. 126 (1967), 514-533.

[Gr] S. J. Greenfield, Cauchy Riemann equations in several variables, Ann. Scuola Norm. Sup. Pisa Sci. Fis. Mat. 22 (1968), 275-314.

[H] G. M. Henkin, The H. Lewy equation and analysis of pseudo-convex manifolds, Uspehi Mat. Nauk 32 (1977), 57-118; Russian Math. Surveys 32 (1977), 59-130.

[Ho] L. Hörmander, An introduction to complex analysis in several variables, Van Nostrand, Princeton, N. J., 1966.

[HP] R. Harvey and J. Polking, Fundamental solutions in complex analysis. I, II, Duke Math. J. 46 (1979), 253-300, 301-340.

[HT] C. D. Hill and G. Taiani, Families of analytic discs in $\mathbf{C}^{n}$ with boundaries on a prescribed CR-submanifold (preprint).

[HW] L. R. Hunt and R. O. Wells, Extension of CR-functions, Amer. J. Math. 98 (1976), 805-820. 
[N] R. Nirenberg, On the H. Lewy extension phenomenon, Trans. Amer. Math. Soc. 168 (1972), 337-356.

[NW] R. Nirenberg and R. O. Wells, Approximation theorems on differentiable submanifolds of a complex manifold, Trans. Amer. Math. Soc. 142 (1969), 15-25. MR 36 \#140.

[T] G. Tomassini, Trace delle funzioni olomorphe sulle sorrovarieta analytiche reali d'una varieta complessa, Ann. Scuola Norm. Sup. Pisa Sci. Fis. Mat. 20 (1966), 31-43.

[W] R. O. Wells, Jr., Holomorphic hulls and holomorphic convexity of differentiable submanifolds, Trans. Amer. Math. Soc. 132 (1968), 245-262.

Department of Mathematics, University of Michigan, Ann Arbor, Michigan 48109

Current address: Department of Mathematics, Texas A \& M University, College Station, Texas 77843 